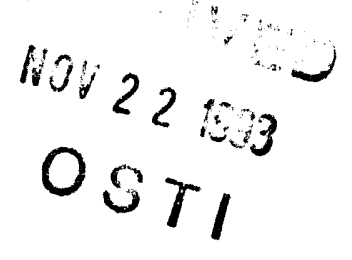

\title{
ELECTRON MICROSCOPE STUDIES
}

\author{
Progress Report \\ DOE ER 60437-6
}

for the period of June 1, 1992 to November 1, 1993

\author{
A. V. Crewe and O. H. Kapp \\ The University of Chicago \\ The Enrico Fermi Institute and \\ The Department of Physics
}

November 1, 1993

Prepared for

The U.S. Dept. of Energy

Agreement Number DE-FG02-86ER60437 


\section{Notice}

This report was prepared as an account of work sponsored by the United States Government. Neither the United States nor the Department of Energy, nor any of their employees, nor any of their contractors, subcontractors, or their employees, makes any warranty, express or implied, or assumes any legal liability or responsibility for the accuracy, completeness, or usefulness of any information, apparatus, product or process disclosed or represents that its use would not infringe privately-owned rights. 


\section{Summary}

During the past year we have continued our work on the mirror-corrected high resolution STEM. We have made significant progress in the design and fabrication of the various microscope sub-systems and have completed a new display system. Additional calculations and computer simulations have been performed to confirm the original theory of mirror correctors.

In our biological work we have made a careful study of the structure of globins, vertebrate and invertebrate, using the accumulated information contained in the Brookhaven Data Bank (3D structures), the Protein Identification Resource (ID sequences) and the data we have obtained with the STEM. Statistical templates have been generated to predict various classes of globins. 


\section{Microscope Development}

\section{Overview}

The principle goal of our microscope development program is to find ways to improve the performance of electron microscopes by correcting the aberrations of the lenses which are used to form the image. In this respect we are the smallest of only three groups in the world who are pursuing such a goal. The other two are the ones led by Gertrude Rempfer in Washington and Harald Rose in Darmstadt, Germany.

In our case we are attempting to design and build a high resolution STEM, whose lens aberrations are to be corrected using an electron mirror in a scheme which we originated and proposed last year.

During the past few months we have confirmed the original theory with a series of lengthy digital computations. These new calculations provided insight into methods of varying the amount of correction. They also confirmed the fact that we do not need to expose the specimen to the high electrostatic fields in the mirror itself. This, of course, is a very important and significant development, since exposure to those fields would generate significant forces on the surface.

We have also completed the design of the electron gun which is needed for the STEM and have begun to design the objective lens. The present status of the gun is that the long lead time components, such as the high voltage insulators, have been ordered, the vacuum vessel is expected to be delivered in a few weeks, and some items are being fabricated in our own machine shop. 
With regard to the objective lens, it appears that we will be able to use the lens coils from a previous project, and possibly the lens housing and pole pieces from that project. We have made contact with microscope manufacturers who may be able to supply a suitable specimen stage. This is of great importance since stage design is an art and the necessary experience does not exist here. We do have a stage which might serve our needs, but one made by an experienced manufacturer would be preferable.

With regard to the electronics, we have built a new display system which is based on the use of a variable speed, high resolution frame store board made by Imaging Technologies. It is driven by the digital scan generator which we designed and built in a previous project. We are now building a digital electronic system to control the electron gun.

One peripheral activity deserves some mention. It appears conceivable that our electron mirror corrector could be used as an in-line corrector if we can use time-of-flight techniques to form an electron trap. We are attempting to confirm this by direct calculation, but these calculations are somewhat difficult. On the assumption that the scheme can be made to work, we have contacted Prof. Alan Feinerman at the University of Illinois here in Chicago. At the UIC they have a microfabrication facility which would be ideal for making the system that would be needed. Perhaps this cooperative venture may have made progress by this time next year. 


\section{The Simple Test System}

Last year we proposed the construction of a simple electron optical system for the purpose of testing the principles of operation of the electron mirror system for the correction of aberrations. We have now built such a system, although it is not yet in operation. Although we called it a "simple" system it has turned out to be mechanically more difficult than we had imagined and for this reason it is probably serving a very good and intended purpose in allowing us to investigate better ways to make a final correction system.

The basic electron optical system is that of putting a small source of electrons into a region of uniform magnetic field, and then as they proceed along the axis of the field they will come to successive foci. We are concerned with a system here which has two successive foci. In the very elementary system of a source and a magnetic field, the distance between the source and the first focus will be equal to the distance between the first focus and the second focus. What we propose to do is to place an electron mirror in the second region so that the electrons will be reflected back towards the source and the second focus will then occur very closely, in the same position as the first foclis. The electron mirror can be turned on or can be turned off according to the operator's choice. When the mirror is turned off the electrons will reach the second focus, and by providing the system with a scanning mechanism one can obtain an image of a specimen placed at that position. When the mirror is turned on the electrons are reflected back towards the position of the first focus where we place an identical specimen and achieve another focus. It is our contention from the theory of this system that the focus with the mirror on will be far better than the one with the mirror off and it is this conclusion which we proposed to test. 
It is obvious that the system is easiest to build when the electron energies are low and, in addition, we should be able to achieve the maximum visible effect of the mirror when the source itself is small. These two factors imply that we should use a field emission source because they are very small and also because they emit electrons of energies of only a few kilovolts which are low enough to be focused quite easily. The use of a field emission source also means that the vacuum level has to be very good because a field emission source can only operate in a very high vacuum environment. Putting all of the factors together we came to the conclusion that the optimum system would be a field emission source operating at a few kilovolts, somewhere between 3 and 5 kilovolts, inserted into a magnetic field of a few hundred gauss so that the electrons would arrive at the first focus in a distance of about $5 \mathrm{~cm}$. These general factors put a scale on the whole test system. It is obviously going to be 10 or $20 \mathrm{~cm}$ long, and since it requires a magnetic field of a few hundred gauss over that length the design of the magnetic field region becomes one of the most important factors.

It is of considerable importance to reduce the radial extent of the system as much as possible. The reason for this is a very simple one: namely that the greater the area over which the magnetic field exists, the greater the amount of iron we must use and the greater the weight of the entire system. Taking everything together we decided that we would attempt to design the entire electron optical system so that it would fit inside a commercially available piece of stainless steel vacuum tubing with conventional vacuum fittings on the ends. The most convenient size that we could find was tubing which fits in a $23 / 4$ in. standard flange and which is about $15 / 8$ in. in diameter. This means that the bore of the magnetic field region must be big enough to accommodate a $23 / 4$ in. flange. Allowing for the coil to go around the outside of that and then 
the iron to go around the outside of that we end up with a system 6 in. diameter or so and 10 or 15 in. long as being the minimum that would satisfy all of these requirements.

The major difficulty in the design was that of providing the uniform electrostatic field which will act as a mirror. We could not do this using a pair of flat electrodes, which is of course the first configuration which comes to mind, simply because the diameter of the tube is small and the axial extent of the mirror region is of the same general size as the diameter. Flat electrodes would therefore not be large enough in their radial extent to provide a uniform field. In order to provide the uniform field it was therefore necessary to use a series of electrodes, each of which is in the shape of an annulus, looking something like a metal washer. These washers, of course, must be separated by insulators and these insulators must be shielded from view from the electron beam. Actually, the more of these electrodes we could provide, the more uniform the field will become, but there is a limit to what we can do here and we allowed for about 8 such electrodes. Each of these electrodes must be provided with its proper potential and therefore each of them must be connected by a wire to a source of voltage. The most logical system is to connect all of these together in series using a chain of resistors and apply one voltage across the entire assembly.

The design problem was that of putting all of this inside the vacuum tube, and also making it possible to put scan coils in the same region in order to deflect the electron beam and ultimately provide an image. It can be imagined that the disposition of electrodes and insulators, and coils and resistors becomes quite complicated and difficult to manage in such a small volume. Nevertheless, we succeeded in working out a design and we put the whole system together.

Primarily because the insulators began to take on complex shapes it became advisable to find a material which would be machineable. The first thing that came to mind was to use 
polyimide, since this has been shown to be very machineable and is often quoted as being compatible with ultra high vacuum systems. For this reason we purchased a block of polyimide and fabricated the entire system using this material. It was indeed eminently machineable, having about the same characteristics as brass.

When the entire system was put together we found that it was impossible to achieve a vacuum level adequate for the operation with a field emission tip, although it was very difficult to estimate the precise vacuum level because the only measure we had of the vacuum was that obtained in the pump itself. The space available in the vacuum tube of the electron optical system was so constrained that it is not possible to put a vacuum gauge in that area. We thought at first that the reason for the poor vacuum was that there is a lot of residual gas in the end of the vacuum tube away from the vacuum pump and we therefore placed a getter system at that end of the vacuum tube. Fortunately we had some non-evaporable getters left over from the previous set of experiments.

When these getters were activated the vacuum level certainly did improve but was far from that which was required for the safe operation of a field emission source. Our next approach was to remove the resistor divider from the vacuum chamber. It should be pointed out that initially we had carefully selected the resistors to be used for this purpose as being ones with a surface coating which was compatible with ultrahigh vacuum. Nevertheless, it was clearly a potential source of difficulty and so we removed the resistors from the vacuum. It should be noted that this is not an easy task since now, instead of having to supply one high voltage feedthrough from the outside world to the inside of the vacuum chamber we now had to supply 8 or 9 
such voltages. Fortunately we were able to find a suitable multi-pin feedthrough from our inventory of vacuum equipment.

Having made this change, the vacuum level certainly improved once more, but still it was not suitable for field emission.

The final suspect was the polyimide insulating material and it was with a great reluctance that we came to this conclusion since the only feasible alternative was to use machineable glass. This latter material is very difficult to work with, especially with the thin walls and precision dimensions that we required for our system. Nevertheless, the change was made, the insulators were remade from this new material, and the system was put together once again. This time the vacuum level in the pump indicated that we had achieved a good enough vacuum level, but unfortunately when the field emission tip was put into operation it became clear that the vacuum level inside the tube was significantly higher than that inside the pump, and although we did achieve field emission, the operating time of the tip was so restricted as to be not worth the attempt to make the entire system operate.

However, it was very clear that the vacuum level would be perfectly adequate for a thermionic field emission source. Unfortunately we did not have such a source at hand and we had to order one. In addition, the use of a thermionic field emission source made it necessary to redesign and refabricate the tip holding mechanism and some of the electronic controls. The reason for this is that the thermionic source requires one additional voltage for its safe operation and the filament must be kept heated at all times, which requires an additional power supply. Since we had to make these mechanical changes, we took the opportunity to rework some of the other components in order to improve the ultimate vacuum level. 
At the time of writing this, the new thermionic source has arrived, all the mechanical changes have been made, and the system has achieved the desired vacuum level. The changes to the electronic system are being made and we will attempt to put the system into operation in the next month or so. 


\section{A High Voltage Electron Gun}

In the design of the proposed STEM it is necessary for the electron beam to pass through the specimen twice. It would be desirable to arrange for the first passage to be through a hole in the specimen. This is not unreasonable, since many specimens are prepared in such a way that there are numerous holes. However, we cannot be certain that this can always be done and we will therefore assume that both traversals are through the specimen. On the first passage the resolution is expected to be quite poor, primarily due to the effect of the spherical aberration of the lens, although there may be additional effects due to defocusing. This depends upon the result of some calculations which are now in progress, but is due to the fact that it may not be necessary to produce a precise focus for the first pass through the specimen. Depending upon the values of the counteracting aberrations, it may be advisable to position the first focus before or after the specimen plane while still maintaining the final focus exactly in that plane.

In any case the electrons must pass through the specimen into the electrostatic mirror region. The effect of the electrostatic mirror is to return the electrons to the specimen in such a way that they are focused onto the specimen. This second focus is expected to be free from aberrations. As the electrons pass through the specimen for the second time they can be detected using an annular detector.

Because of this double passage of the electrons through the specimen it is clear that we need a reasonably high accelerating voltage. In our previous microscope the accelerating voltage was in the 30-50 kv region, but in that case the number of scattered electrons was around $5 \%$ for the very thin specimens that we used there. For two traversals we would expect about $10 \%$ scattering, which is on the borderline of being useful. One would therefore expect that the 
minimum accelerating voltage that we can use would be about $50 \mathrm{kv}$, although $75 \mathrm{kv}$ would be better and perhaps $100 \mathrm{kv}$ may be aćvisable.

All the electron guns that we have used in the laboratory have been in the $35 \mathrm{kv}$ region, but unfortunately they are an integral part of the entire microscope and it is not possible to use them for the kind of purposes that we now need, and we therefore need a new electron gun.

There are no high voltage, field emission electron guns available on the market and to our knowledge there is only one electron gun with a field emission source which can be purchased as a unit, and that is the electron gun manufactured by FEI (Field Emission Incorporated). However, this electron gun is limited to about $25 \mathrm{kv}$ and in addition it uses a thermionic field emission source (TFE) which is not very well suited for our purposes, for reasons which will be discussed below. There are no cold field emission (CFE) guns on the market at any operating voltage.

One obvious thought is to ask one of the manufacturers to supply us with such a gun, but there are several reasons for not doing so.

Hitachi has sold many instruments with a $30 \mathrm{kv}$ CFE gun and has built several high voltage CFE guns for internal purposes. In addition they have recently offered a $100 \mathrm{kv}$ gun for use with a TEM. Unfortunately this appears to be a two electrode system, (see later) which does not have the flexibility which we may need.

Several other manufacturers offer instruments with a $30 \mathrm{kv}$ TFE gun similar to that made by FEI, but this is the wrong type of field emitter for our purposes, a technical point which needs further explanation. 
There are two kinds of field emission source, namely cold field emission and thermionic field emission, and there are some substantial differences between these two. The cold field emission source (CFE) operates in a vacuum of $10^{-10}$ Torr and is very sensitive to that vacuum level. However it does have the considerable advantage that the effective source size is very small, namely about $10 \AA$ This very small source size means that one needs very little demagnification in the objective lens in order to provide a small, focused probe. In fact it is quite possible to design a microscope which uses only an electron gun and an objective lens without the need for any additional lenses. The thermionic field emission source (TFE), on the other hand, has the advantage that it can operate at a vacuum level of $10^{-9} \mathrm{Torr}$, which of course makes the design of the vacuum system considerably simpler. On the other hand, the source size is quite large, approximately $200 \AA$, and this means that one cannot obtain a small enough probe for our purposes by using only one lens. If we were to use a thermionic source, we would need to have an electron optical column consisting of the electron gun, a condenser lens, and the final objective lens. This is obviously more difficult to make and more expensive. For this reason then, we select the cold field emission source as being the most appropriate one for our purposes.

The cold field emission source (CFE) has proved to be difficult to engineer into an electron gun. The very first of these electron guns was the one that we developed almost 30 years ago, and that was the Butler gun which has 2 electrodes: the tip itself, an extraction electrode, and the final anode. Two voltages are applied to the gun, $V_{1}$, which is the extraction voltage applied between the tip and the first anode, and $\mathrm{V}_{0}$, which is applied between the tip and the final anode. In operating the gun, $V_{1}$ is adjusted in order to obtain the desired electron current and $V_{0}$ is adjusted to obtain the desired accelerating voltage. 
There are two features to be noted here. One is that the electron current is an extremely sensitive function of $V_{1}$. It is at least exponential in its dependence. A very small adjustment to $V_{1}$ can produce a very large increment in the beam current, and in fact it is very easy to destroy the field emission tip by demanding too much current from it. The other feature to be noted is that the electron gun constitutes an electrostatic lens, and the focusing effect of this lens depends upon the ratio of $V_{0}$ to $V_{1}$. Since one selects these two voltages independently, this means that the focusing effect of the gun can vary significantly for different settings of these two voltages, and this makes it somewhat difficult to design a complete electron optical system. In the case of the original STEM we were constrained by this effect to operating at around 30 or $35 \mathrm{kv}$ simply because the field emission sources themselves operated at about $3 \mathrm{kv}$. This was a cause of considerable inconvenience. For example, as the field emission tips got older and blunter their operating voltage would rise, and in order to keep the same focusing properties of the electron gun, it was necessary to raise the accelerating voltage of the entire microscope. This could only be done within certain limits.

There is another, more subtle difficulty with this Butler gun and that is the question of electrical discharges. Both the tip and the first electrode are at a high voltage with respect to their surroundings, and there is always some possibility that there could be an electrical discharge to ground. If this should occur from the first anode, then the tip itself can be exposed to the accelerating voltage, and as we mentioned before, the emission current is a very sensitive function of the applied voltage, and so the tip will destroy itself. The Butler gun therefore has the considerable disadvantage that even a small electrical instability can cause the loss of the field emission tip. Since we need an even higher accelerating voltage for our new application, this 
increases the sensitivity to electrical discharges. It should be noted that it is far more difficult to eliminate discharges for a system operating at say $50 \mathrm{kv}$ than it is at $30 \mathrm{kv}$. Discharge problems are a very sensitive function of the applied voltage.

Both these effects can be reduced or eliminated with the use of a 3 electrode system, that is to say the tip, a first anode which defines the beam current, a second anode, which can do some focusing, and then the final anode. We apply a voltage $V_{1}$ between the tip and the first anode, a voltage $V_{2}$ between the tip and the second anode, and a voltage $V_{0}$ between the tip and the final anode. This type of gun allows the control of the focusing action of the gun by adjusting the value of $V_{2}$. The gun will focus when $V_{2}$ is either greater than or less than $V_{1}$, but it is far more convenient to make it less than $V_{1}$ because it provides a greater range of control. If one makes it greater than $V_{1}$ it may have to be substantially greater in order to provide the focusing action. In any case, with this electrode system it is possible to choose $V_{1}$ in order to obtain the electron current that is needed, $V_{0}$ in order to select the accelerating voltage, and $V_{2}$ in order to provide an external focus of the electron beam at any required position. This then allows us to use a single objective lens in order to produce the final focus on the specimen.

It should also be noted that this structure also makes it possible to provide some protection against electrical discharges. For example it is possible to surround the tip in the first anode with a cup shaped electrode to which the voltage $V_{2}$ is applied. In this case any electrical discharges are likely to take place from $V_{2}$ to ground, and then the only effect on the tip would be some possible capacitative coupling, which would temporarily increase the emission current. While this protection is far from complete, it is better than none. 
This design of electron gun might very well be adequate for our purposes, but it is not entirely clear. This design has been used by several different microscope manufacturers and it is the basis of the FEI gun, but to our knowledge none have been operated above about $30 \mathrm{kv}$. We need to go considerably higher than this, and then the risk of electrical discharge becomes very much higher.

As we noted above, the probability of encountering a discharge is very much greater at say $100 \mathrm{kv}$. than it is at $50 \mathrm{kv}$. Naively one might think that by increasing the size of insulators or the gap between conductors by a factor of 2 , that we could easily transform a $50 \mathrm{kv}$ design into a 100 kv design. Unfortunately this is not true. The discharge problem goes far more rapidly than simple linear scaling.

In considering this problem we decided that one possibility was to add yet one more electrode to the system. By placing an electrode between the focus electrode and the final one, we can apply a voltage which is intermediate between $V_{2}$ and the final electrode, which is ground. In addition, we can put a cylindrical shield around the other electrodes, with the shield being connected to this intermediate one.

Suppose for example we want to operate at $100 \mathrm{kv}$, we would apply $50 \mathrm{kv}$ to this intermediate electrode, and the $100 \mathrm{kv}$ of course would be applied to the tip and approximately $100 \mathrm{kv}$ to the first anode and the focus electrode. Then the maximum potential difference that occurs anywhere in the system would be $50 \mathrm{kv}$, and not $100 \mathrm{kv}$. The effect of this would be to significantly reduce the probability of an electrical discharge, at the same time the focusing of the gun would be very little affected. 
These various configurations of electrodes are indicated in Figure 1. In Figure 1a. we show schematically the Butler gun, consisting of the tip and two electrodes. We also indicate the two voltages which are applied to the system. Note that the final electrode is normally connected to ground. In Figure 1b. we show what is called the 3-electrode gun, where we indicate the additional voltage $V_{2}$, which is applied to the focus electrode. In Figure 1c. we indicate our proposed system whereby an additional electrode is placed between the focus electrode and ground, and the voltage which is applied to that electrode can be obtained very simply using a potential divider from the $V_{0}$ power supply. We would propose that this potential be a fixed ratio of $\mathrm{V}_{0}$, almost certainly exactly one half.

A more schematic arrangement of the proposed electrode system is shown in Figure 2. Here we indicate the two connections which must be made to the tip to provide for the flashing current, the two electrodes which comprise the extraction voltage and the focus voltage, and the intermediate electrode which is now shown to have an electrostatic shield around the gun elements. It is this shield which provides a protection and which prevents the full value of $V_{0}$ being applied as a potential difference to ground. It should be noted that we can also make the electrostatic shield out of a magnetic material, so that it is also a magnetic shield. Finally, the outer vacuum envelope is indicated, and this also can be made of magnetic material, thereby providing a double magnetic shield to isolate the electron beam from the stray magnetic fields which exist in any laboratory.

With regard to the actual geometric design $r^{r}$ he electrodes, it may be recalled that in the original Butler gun the shape of the electrodes was a salculated one which provided the simplest possible potential distribution between the extraction electrode and the final electrode. The 
general idea behind that design was that the potential gradient, that is to say the electrostatic field, should be zero in passing through the two apertures. Using this condition the apertures themselves do not focus the electron beam, and all the focusing is done by means of the shapes of the electrodes ther .elves, and those shapes can be carefully controlled. In the older design of gun all the apertures in the system can act as focusing elements, thereby giving an element of uncertainty in the design of any gun, but in the Butler system this problem is successfully avoided.

In later calculations, we have satisfied ourselves that the crucial factor in the design of the electrodes is that the shape near to any aperture should be such as to provide a zero field at the aperture. This is best done when the electrodes are tapered at an angle whose tangent is $\sqrt{2}$.

This angle which is about $57^{\circ}$ is closely approximaied if we cut a taper of $60^{\circ}$ near to any aperture. In other words, the Butler gun can be very closely approximated by using two reasonably thick electrodes and cutting a taper into the electrodes at an angle of $60^{\circ}$ at the position of the apertures. This design is virtually indistinguishable from the Butler design and is far simpler to construct.

In the case of our 4-electrode system, we only need to taper the apertures in the region near to the first aperture, that is to say the one in the extraction electrode and the aperture at the exit of the gun. The reason for this is that we have found that the apertures in the central electrodes are best left quite large, and being large, they have only a small effect on the field along the axis. This has the additional feature that the alignment of these electrodes is not very critical. Taking all these factors into account, we can now proceed with the design of the actual gun. 


\section{Mechanical design of the gun}

In Figure 3 we illustrate the major features of the gun design. The vacuum vessel is 6 in. in diameter, and the high-voltage connection will be made through the bottom since we have decided to use electrons moving upwards rather than downwards. The vacuum vessel contains an inner electrode which is separated from the main vessel by two insulators in the form of quartz rings, $11 / 2$ in. long. The inner conductor is in the form of a smooth cylindrical can, and as we mentioned before, it will be raised to one half the total voltage. That is to say if the accelerating voltage for the electrons is to be $100 \mathrm{kv}$, then $50 \mathrm{kv}$ will be placed on this inner cylindrical electrode. Therefore the only voltage to ground is 50 kilovolts, which we believe can easily be maintained with a gap of about $1 \mathrm{~cm}$., which is indicated in the drawing. With regard to magnetic shielding, there is a mu-metal cylinder just inside the vacuum vessel wall, and the inner conductor will also be made of mu-metal so that we have double shielding. In order to get a good vacuum, each of these mu-metal shields will be perforated.

The inner structure of a gun is illustrated in Figure 4, and here we see the details of the contents. This inner conductor is a can containing a system of electrodes which are self-contained so that everything is one rigid unit. The position of the tip is indicated, and following the tip there is an extraction electrode (8), the focus electrode (9), and the intermediate electrode (10). The total voltage of 100 kilovolts would be applied to the tip, and the extraction voltage, which is only 3 or 4 kilovolts, is applied between the tip and the extraction electrode. There is only a small voltage of perhaps 1 or 2 kilovolts between the extraction electrode and the focus electrode, so this only requires a small insulator, which is the quartz ring (7). However, there is a large voltage of about 50 kilovolts between the focus electrode and the intermediate electrode, and similarly 
between the focus electrode and the outer vessel, and this requires additional quartz rings (2). In this particular design, then, we have an electron gun in the form of this inner cylinder, which can be preassembled into a rigid unit. This unit can then be inserted in the vacuum can to form the complete electron gun. We propose that the electrode alignment would be done mechanically prior to assembly. The reason for this is that the apertures in the focus electrode and the intermediate electrode are quite large, and because of this they are not very sensitive to alignment. The outer can (3) is welded to the end rings (1) and the internal components are held in place by means of a set of springs (5).

The most critical alignment procedure will be that of ensuring that the tip itself is on the axis of the gun. We plan to do this by allowing for a small adjustment at the bottom of the gun. The high-voltage electrodes will be connected to the vacuum flange (20), which in turn is mounted on to an 8 in. flange (21), which is connected to the base of the vacuum vessel (23) by means of a flexible bellows (22). These two large flanges in the bellows will be assembled as one unit, with a differential screw arrangement to allow for a small tilt between the two flanges. By using a differential screw we can make very fine adjustments and perform the alignment of the gun.

With regard to the vacuum system of the gun we plan to use a small ion pump which will be connected to the outer vacuum vessel (24) at right angles to the axis of the diagram. In addition to this we will probably use 1 or 2 non-evaporable getters in order to achieve the necessary vacuum. We believe that with the careful choice of materials and attention to the cleaning process that we should be able to achieve the required vacuum level with a pump on the order of 20 liters/sec. 


\section{The Objective Lens}

The essential problem of designing our new mirror corrector is that the magnetic lens tends to become physically very large, and the design problem is that of trying to minimize the size of the lens in order to reduce the cost and the weight of the whole system. Perhaps this difficulty will be become more evident as this description proceeds.

The objective lens of the new microscope is an unusual one and it presents the designer with problems which have never previously been encountered in a magnetic lens. The reason for this, of course is that we must introduce an electrostatic mirror into the magnetic field region. This means that we have to introduce some form of high voltage terminal into the magnetic field, and this presents us with both magnetic and electrostatic design problems. There will also be some new problems in connection with the vacuum system but we intend to investigate these after the magnetic design is completed.

The voltage that we must introduce has to be at least equal to the accelerating voltage of the microscope, and in our case this might be as high as 75 or $100 \mathrm{kv}$. It can be appreciated that the insulators for such a high voltage terminal are necessarily quite large, and the spacing between the electrodes must also be quite large, and it is these two factors which make the design of the lens quite difficult.

In reviewing possible designs it would seem that there are two basic design options and we must choose one of them. The first and perhaps most obvious approach would be to introduce a separate mirror electrode between the poles of the magnet. The second alternative would be to electrically isolate one of the poles so that the magnetic pole itself can also act as the electrostatic mirror. 
We look at the first alternative, which is illustrated in Figure 5. One can see that if we were to adopt this design we must provide a large opening in the magnetic shroud for the introduction of the high voltage mirror electrode. This is of course feasible, but on the other hand, it also introduces substantial asymmetry into the magnetic field and it is somewhat doubtful whether that asymmetry could be entirely removed from the region of the electron beam. One further difficulty is that the high voltage terminal requires space on both sides to prevent discharges. For example, we might require a space of $1 \mathrm{~cm}$ or so between the specimen and the mirror electrode because $1 \mathrm{~cm}$ is about the smallest gap that we would like to work with, considering the value of the high voltages. Similarly, we would need a gap of $1 \mathrm{~cm}$ between the mirror electrode and the upper lens pole piece, and this makes it quite clear that the introduction of a separate mirror electrode would mean that the gap between the poles would be large and because of this we would require a greater lens excitation. This is unfortunate because we would be providing field strength in a region where the electrons never go. This in turn requires more power to be dissipated in the coils, which forces the lens to be larger and the whole structure of the lens begins to become excessively big.

The alternative design is to isolate the pole piece and this is illustrated in Figure 6 . In this case the upper pole itself acts as the electrostatic mirror, and we must provide electrical isolation between the pole piece and the magnetic shroud. However, this does not appear to be difficult to do since the radius of the pole piece at that point can be quite large and this reduces the electrical stress so that the insulator can be thinner. In addition, the magnetic design of the lens is not seriously impaired. The gap can now be smaller by $1 \mathrm{~cm}$ than the previous design, and the effect of the gap between the pole piece and the shroud can be made small by increasing the thickness of 
the shroud and increasing the radius of the insulator. By careful design, the magnetic reluctance of the insulator gap can be made small with respect to the reluctance of the gap between the pole pieces. By doing this we can reduce the number of ampere turns required for the excitation of the lens so that this particular design can be made smaller than the design illustrated in Figure 1. However, the penalty we pay is that the insulator itself becomes a difficult design problem.

In both the designs of Figures 5 and 6 we have some serious problems with regard to the design of the vacuum system, but for the moment we will set these on one side.

The entire design of the lens is conditioned by the need to provide a sufficiently large gap to prevent electrical discharges between the mirror and the rest of the system. There are no hard and fast rules with regard to the size of this gap and the problem is made a little more difficult by the fact that we will almost certainly need to work with an ultra-high vacuum system. We plan to base our choice of the size of the gap on the experience we have already had with regard to electron guns. In that case we have found that we can safely maintain a voltage of $30 \mathrm{or} 40 \mathrm{kv}$ across a gap of $3 \mathrm{~mm}$ and from this it would appear reasonable that for a high voltage in the range of 75 or $100 \mathrm{kv}$, we would be comfortable with a gap of perhaps 8 or $10 \mathrm{~mm}$. Certainly we would not wish to go very much smaller than this for our first attempt at a design. If we assume now that we can use a gap of $1 \mathrm{~cm}$, we can look at the theory of the electron mirror, where we find that the length of the mirror region is given by

$$
l=\frac{\pi}{2 U}
$$

where the parameter $U$ is one that we have used in several publications. It is the ratio of the magnetic field to the magnetic rigidity and is related to the accelerating voltage by the equation

$$
U=\frac{B}{3.37 \sqrt{V}}
$$


If we accept this condition then we calculate the value of $U$ at about 1.57 and so tentatively we take the approximate value $U=1.5 . \quad \mathrm{U}$ is a universal parameter and has the dimensions of inverse length, and if we measure the magnetic field in gauss and the rigidity in gauss centimeters, then $U$ is given in units of inverse centimeters. We can then make a rough estimate of the magnetic field involved by assuming a magnetic rigidity of 1000 gauss centimeters, which corresponds to an electron of around $100 \mathrm{kv}$. Using a value of $U=1.5$, we arrive at a magnetic field value of 1500 gauss, a field which is quite low compared to the fields which are normally used in objective lenses. This means that the focal length will be considerably larger than in a normal lens and also the aberration coefficients will be large.

We can now also estimate the amount of spherical aberration correction which such a mirror can introduce because that is also given by the theory of the mirror and we have

$$
C_{s}=\frac{2 \pi}{U}
$$

so that we see that with a gap of $1 \mathrm{~cm}$ which we have allowed for the electrical isolation then we can introduce $4 \mathrm{~cm}$ of spherical or chromatic aberration correction.

For the next step we would like to estimate the amount of spherical or chromatic aberration which is introduced by the magnetic field of the objective lens as the electrons are coming towards their first focus. In order to do this we make some assumptions about the shape of the magnetic field. This is not too difficult to do since the basic structure of the magnetic field region is that of two parallel pole pieces, one of which has a small aperture through which the electrons enter. We can very easily approximate the shape of this field, which is given by

$$
U(z)=\frac{U}{\pi}\left[\frac{\pi}{2}+\arctan \left(\frac{z}{A}\right)\right]
$$

This is a very reasonable approximation and is quite often used for electrostatic fields. In this case since the iron is far from the saturation region, the expression will also apply for the magnetic field 
and in any case the spherical aberration of such a magnetic field is not drastically dependent upon the details of the shape. We only really need to know that the magnetic field rises from zero to some constant value in a smooth fashion.

In order to calculate this we have done some ray tracing analysis, the results of which are shown in Figures 7 and 8 . In Figure 7 we indicate that we have fixed the value of $U$ at 1.5 and then calculated the position of the focus, that is to say the first electron focus, as a function of the size of the aperture in the pole piece, and we see that the focal position is not drastically dependent upon the size of the hole, which is to be expected. Most of the focusing is done by the uniform magnetic field region. The excitation of the lens is given as the ratio of the ampere turns to the magnetic rigidity, and it too is roughly constant. The gap length, which is also indicated in Figure 7, is very simply the focus position plus $1 \mathrm{~cm}$, which is the distance we have allowed for the electrostatic mirror. One san see then that the gap between the pole pieces is going to be around $3.5 \mathrm{~cm}$. Turning to Figure 8 , here we indicate the calculation of the spherical aberration coefficient of the magnetic field region. As is always the case, the spherical aberration of the magnetic field region is negative. It is quite large tor the case of small apertures, which is entirely to be expected from the elementary theory of magnetic focusing, and reaches a value of around 1 $\mathrm{cm}$ for a large aperture. In comparing this with Figure 7 one can see that the ratio of the spherical aberration to the focal position is about $1 / 2$, which again is entirely to be expected from the theory of magnetic lenses. In view of this we feel then that we understand this magnetic focusing and have some faith that the calculations are correct. In order to calculate the total spherical aberration constant we take the spherical aberration of the lens and add 4 times the gap, namely 4 $\mathrm{cm}$, and one can see then that the total spherical aberration of the system passes through zero at a 
gap of about $.05 \mathrm{~cm}$. The spherical aberration is negative for smaller apertures and positive for larger apertures.

We still must add to this calculation the aberrations of the rest of the system, that is to say we have to include the electron gun and perhaps a condenser lens. These aberrations would both be negative and would therefore shift the crossover point towards larger apertures.

From this preliminary analysis we can see that it is indeed possible to design a system which has zero spherical aberration. The only problem we are left with at the moment is precisely how to go about setting this zero point. At first sight it might appear that we should aim for the one which is indicated in Figure 8, that is to say we ought to adjust the aperture in order to find the position of zero aberration. However, it is extremely difficult to adjust the aperture in the lens and as a practical matter it cannot be done.

In this respect it is perhaps important to review one of the calculations we have done previously. This is a publication which has not yet appeared but should appear soon. These particular calculations were done with this problem in mind and are therefore directly relevant. The aim of the calculations was to try to understand the way in which a practical mirror system would function. For example, the specimen is at ground potential and on one side of the specimen everything else is also at ground potential while on the other side there is a strong electrostatic field. This would tend to apply forces on to the specimen, which could be extremely disruptive. In addition, in order to keep the electrostatic region well controlled and uniform it would be necessary to imbed the specimen into a large, flat, conducting surface. This is obviously quite impractical. A better scheme would be to have a large flat conducting surface with a hole in it for the electrons to pass through and the specimen positioned below the aperture in a region 
where the electrostatic field is very small. We fully expect that this displacement of the specimen would change the aberration properties in the system, and in particular would reduce the correcting action by some amount. It is this point which was studied in the publication. The calculations clearly indicate that by moving a specimen up and down in the region below the electrostatic field aperture we can vary the aberration correction significantly. That is to say, we can always find a point zero aberration.

Taking this into account, then, it would appear that instead of using the zero crossing in Figure 8 we would be better off using a more stable region, such as an aperture with a value of 0.3 or 0.4 . In this region the properties of the mirror are quite stable, there is a significant over correction so that the net spherical aberration is positive, and then by moving the specimen down towards the lower pole piece we can find a region of zero spherical aberration. This provides us with a degree of control which we would not have had if we had chosen to work at the zero point crossing of $\mathrm{A}=.05$.

These are the preliminary considerations which we have given to the design of the objective lens, and we take these to be confirmation that the system can be made to work. We intend to perform much more detailed calculations in the very near future. 


\section{An In-Line Aberration Corrector}

\section{Introduction}

In some previous publications we have developed the theory of operation of magnetically focused electrostatic mirrors and shown how they can be used to correct spherical and chromatic aberration. Specifically, such mirrors can be used to introduce aberrations which are opposite in sign to those of a normal electron lens.

Various geometrical arrangements are possible with this kind of mirror, but most of them suffer from the difficulty that the useful electron beim is moving in the opposite direction to the incident one and it is difficult to separate the two beams without introducing new aberrations. It is true that some configurations can be used where the useful beam is moving in the same direction as the incident beam and it is entirely possible that such arrangements could prove to be practical. However they all require a significant lateral beam displacement and this may be difficult to control with the necessary precision.

There is another possibility for the use of these mirrors which we are now investigating, and that is to use time-of-flight techniques to produce an electron trap inside a mirror. This can be done by inserting "switches" into the column. In principle such a technique could allow a controlled number of reflections and by using an even number of reflections we would have an in-line system for the introduction of reverse aberrations.

The feasibility of such a concept depends upon the availability of electronic devices of appropriate capabilities. In order to proceed with the calculations we will assume here that it is possible to produce a voltage pulse of the order of $10 \mathrm{v}$ in height and with a sub-nanosecond rise 
time. Such devices are generally available and this performance is well within the range of conventional technology.

We are presently investigating various various geometrical arrangements to accomplish all this. At the moment we are not too optimistic. The principal difficulty arises from the need to slow the electron beam down to 10 volts or so. All decelerating lenses have short focal lengths and tend to produce a focus very near to the exit of the lens. This causes difficulties with the first order optics of the system and at the moment we do not see any way clear of the difficulty. Nevertheless the concept is important and we will continue to work on it. 


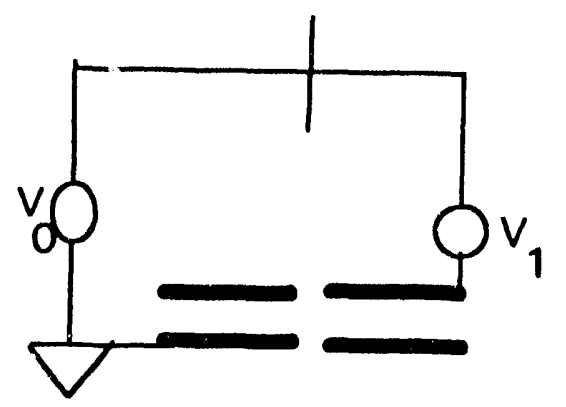

a.

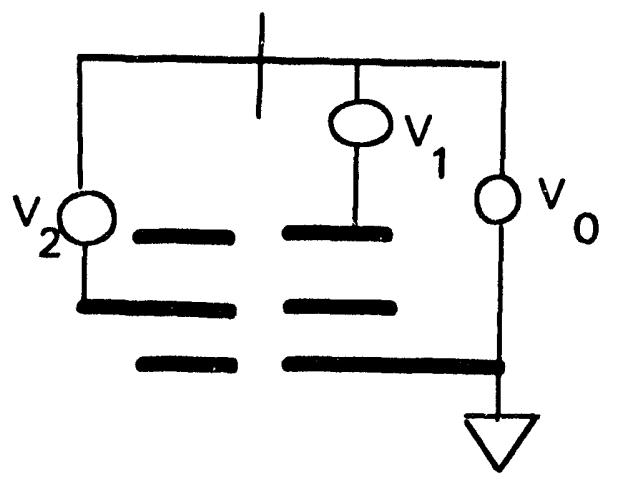

b.



c.

Figure 1: see page 15 for description 


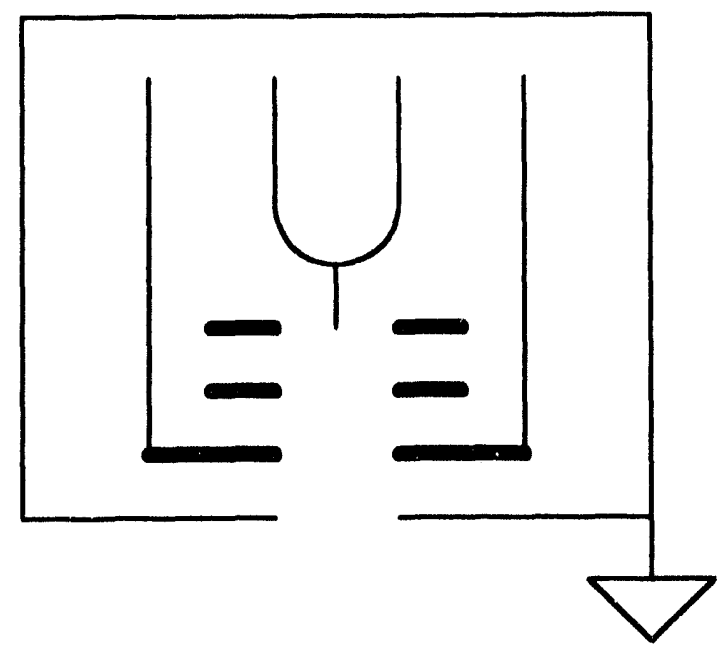

Figure 2: see page 15 for description 


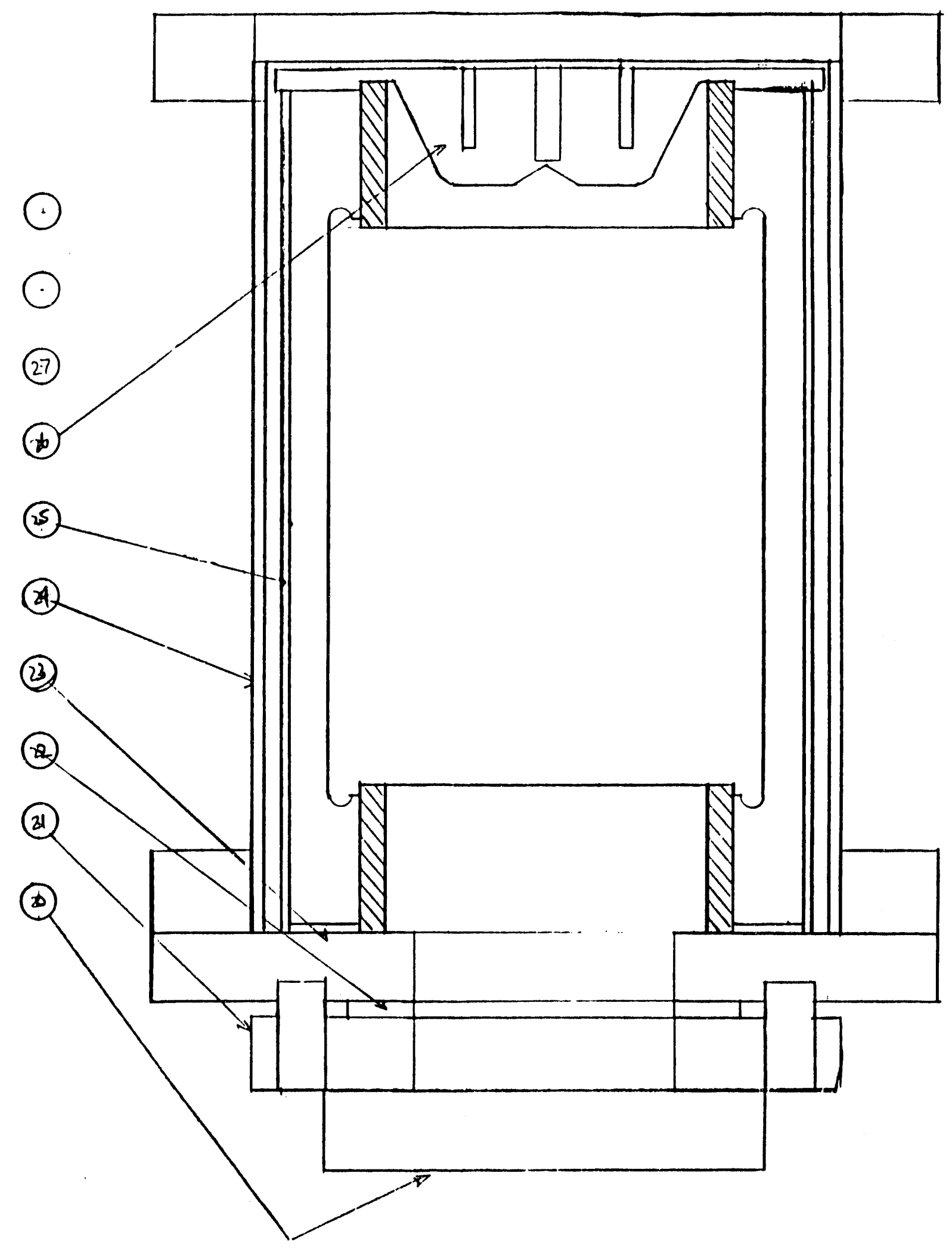

Figure 3: see page 17 for description 


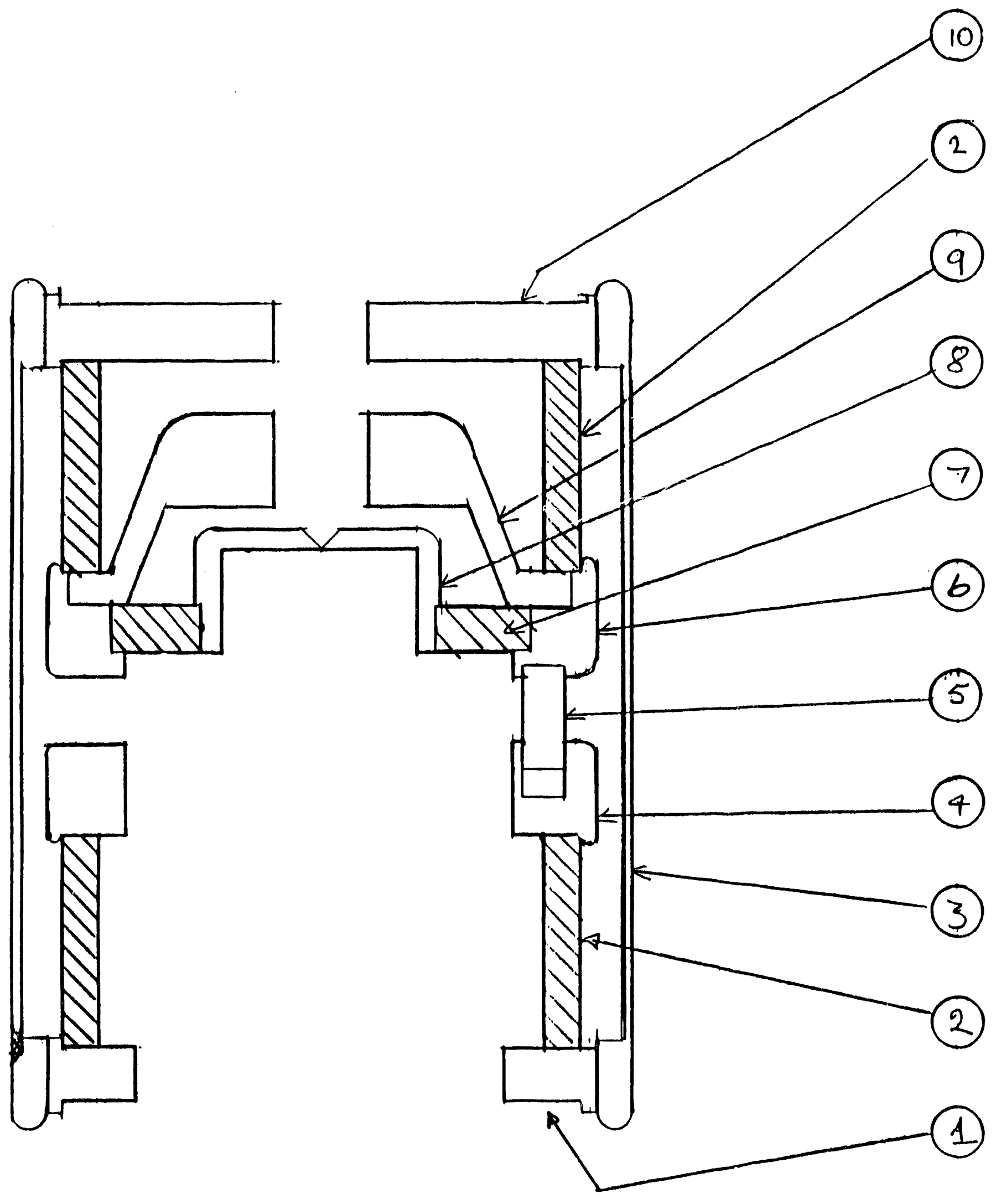

Figure 4: see page 17 for description 


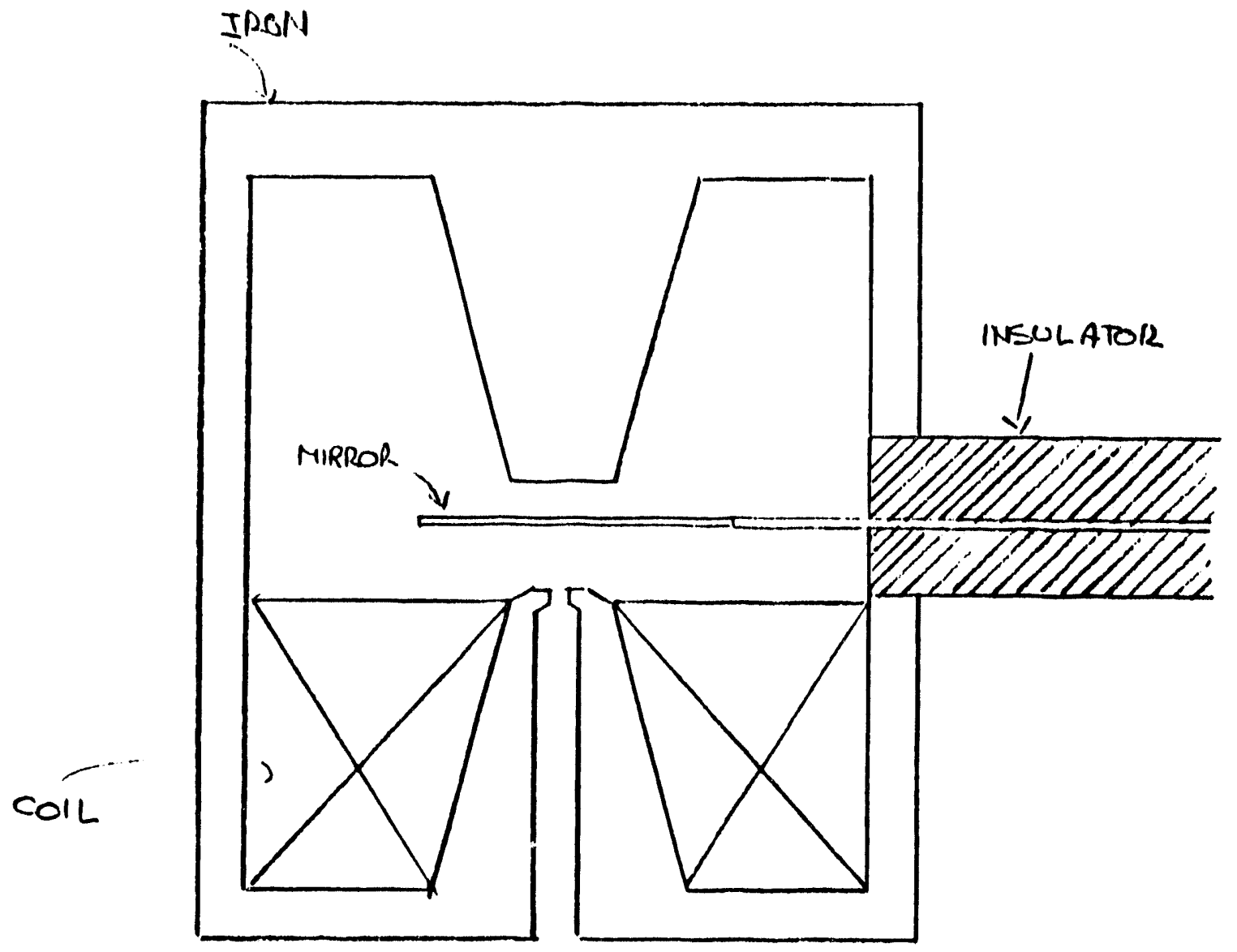

Figure 5: see page 20 for description 


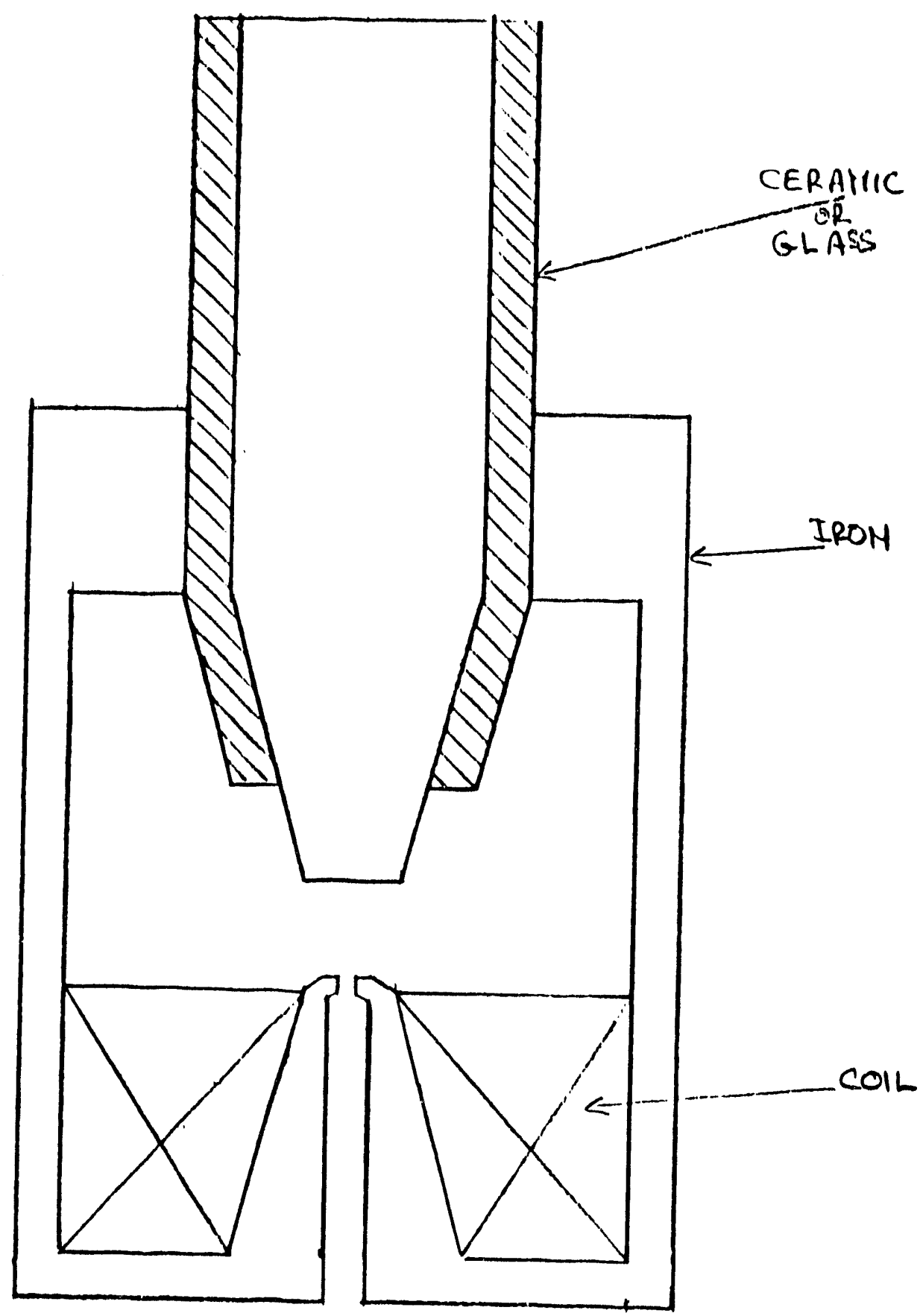

Figure 6: see page 20 for description 


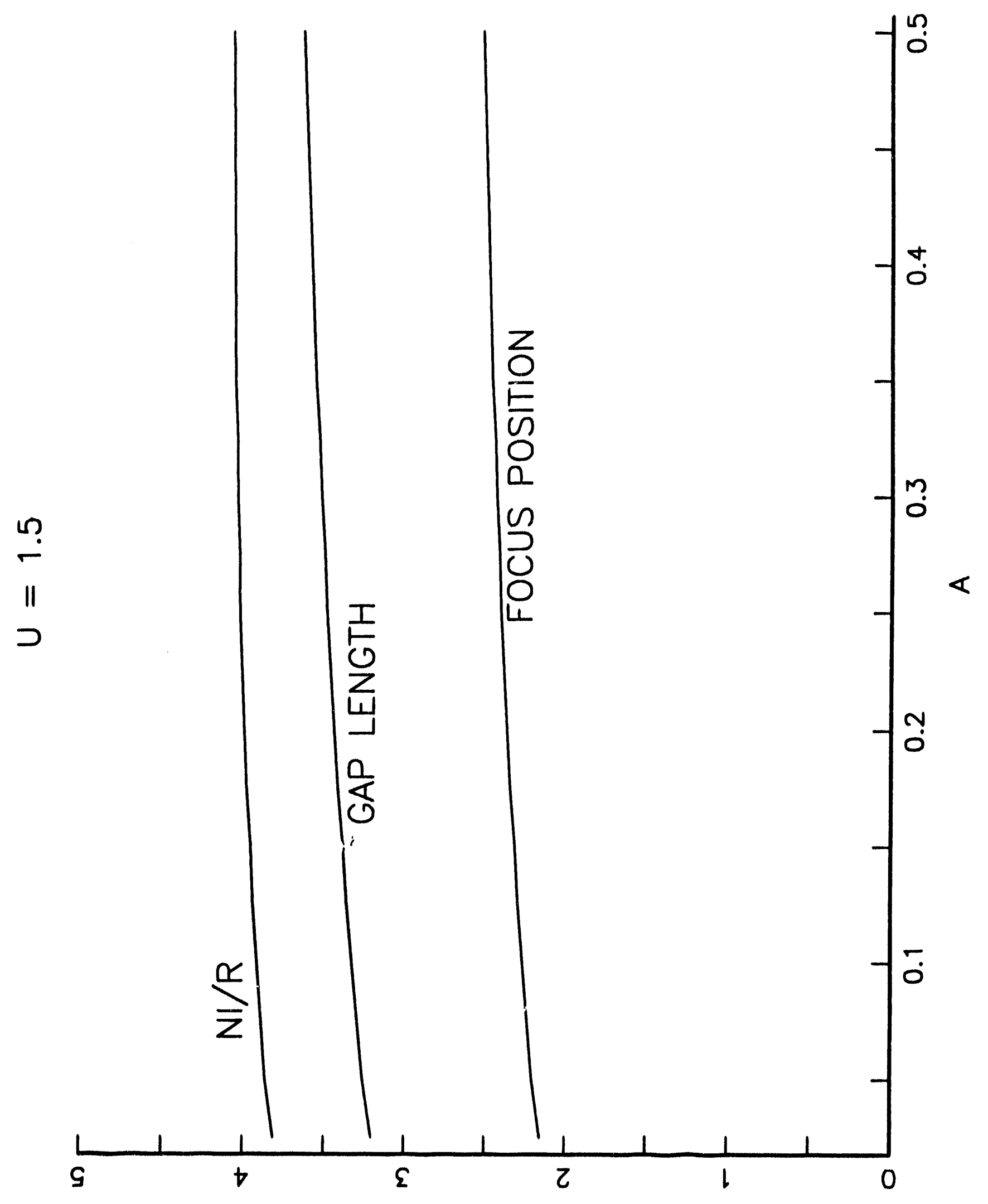

Figure 7: see page 23 for descritption 


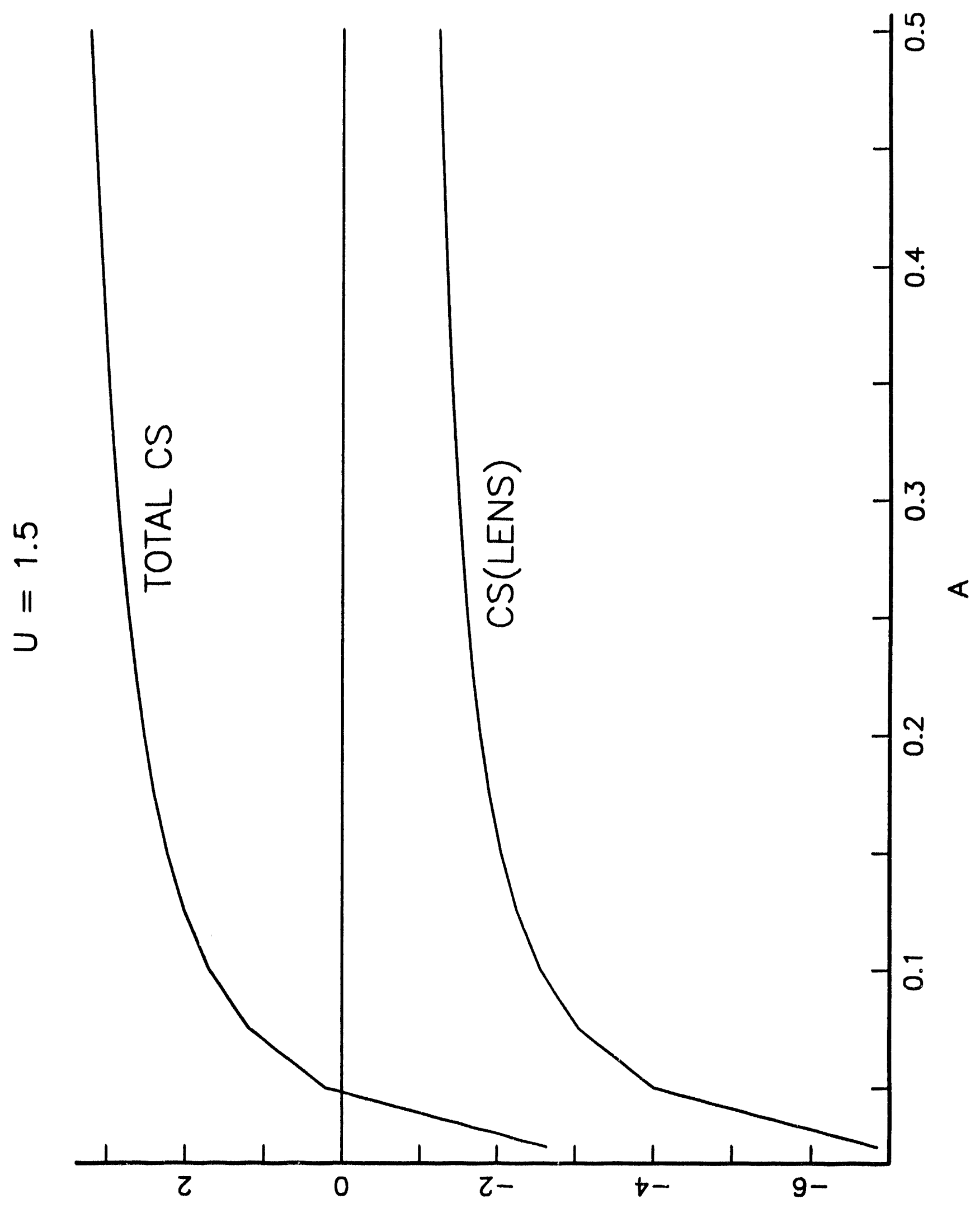

Figure 8: see page 23 for description 


\section{Biological Studies}

\section{Summary}

The unique feature of monomeric globins, polypeptide chains of 132 to 161 amino acids binding one heme group, is the conservation of a characteristic secondary structure, the "myoglobin fold", consisting of 7 or $\mathbf{8}$ alpha-helical segments, in globins of very different origins whose amino acid sequences have $<20 \%$ residue identity (A. M. Lesk \& C. Chothia, J. Mol Biol. 136, 225-270, 1980). The $>1000$ entries in the PIR Data Bank, were checked for unique and reliably determined sequences. Some 554 vertebrate sequences were divided into alpha-like and beta-like chains and myoglobins were aligned usiig the secondary structures from the known crystallographic structures. A total of 142 nonvertebrate globin sequences were found to consist of 116 monomeric globins, 6 truncated globins with less than 130 amino acids and 22 heme-binding domains from chimeric chains containing two or more different, globin-like domains or one globin-like domain and an unrelated protein. The nonvertebrate sequences were aligned using the secondary structures found in the 7 known crystal structures of monomeric nonvertebrate globins. Although 171 positions are sufficient to align the vertebrate globins, with 105 positions common to $99 \%$ of the sequences, 182 positions are needed to accommodate the alignment of all the globins, due primarily to insertions in the interhelical regions A-B, E-F, and F-G. Of the 182 positions, 96 are common to $99 \%$ of the 554 vertebrate and $99 \%$ of the 116 monomeric, nonvertebrate globins sequences. Only two residues, the proximal HisF8 and PheCD1 are conserved in all the sequences. Templates were constructed, based on a statistical analysis of residue occurrence at specific positions within the alpha-helical segments of vertebrate and nonvertebrate, monomeric globins and applied towards the alignment of truncated globins and 
the globin-like domains of chimeric proteins whose sequences have come to light recently. Although our analysis extends and refines the seminal work by Bashford et. al. (J. Mol. Biol. 196, 199-216, 1987), based on 226 amino acid sequences (of which only about $10 \%$ were of nonvertebrate origin), its conclusion differs in suggesting that there exists a continuum of heme-binding, globin-like domains exhibiting some but not all of the features required by the templates, linking the members of the "true globin" family with other families of heme-binding proteins. 


\section{Introduction}

The globin family has a venerable lineage of research dating to the 1960s. The amino acid sequence of sperm whale myoglobin and the human hemoglobin alpha and beta chains were among the first proteins sequences to be determined. Likewise, myoglobin was the first protein structure to be determined by x-ray diffraction (Kendrew et al., 1960), followed soon by hemoglobin (Perutz, 1970). As the number of known globins sequences and crystallographic structures has grown the unique features of the globin protein family have slowly emerged. The early studies focused upon the identification of the sites in the interior of the globin molecule occupied by residues with hydrophobic side-chains (Perutz et al., 1965; Ptitsyn, 1974). In addition, Lim \& Ptitsyn (1970) showed that the total volume of the hydrophobic residues occupying the globin interior remains constant. When the first nonvertebrate globin structure was solved, that of Chironomus hemoglobin (Huber et al., 1970), it was shown that it shared with the vertebrate globins some 15 residue to residue contacts between the interior "core" positions (Kozitsyn \& Ptitsyn, 1974). Furthermore, comparison of the three-dimensional structures of sperm whale myoglobin and the insect globin showed that although the individual helical segments in the two structures were superimposable with a mean difference in atomic position of $\sim 0.5 \AA$ angstroms, the ensemble of the helices taken together, could not be superimposed any better than with a mean difference of $\sim 1.25$ angstroms (Huber et al., 1971). A landmark study by Lesk \& Chothia (1980) of the correspondence between primary, secondary and tertiary structures of several globins provided the first detailed alignment, position by position, of vertebrate globins, sperm whale myoglobin, human and horse alpha and beta chains and lamprey hemoglobin with three invertebrate globins: Chironomus, Glycera, arı annelid, and a plant globin Lupinus leghemoglobin. This study established that some 59 residues are the principal determinants of the 
globin structures and that the "myoglobin fold," consisting of 7 or 8 alpha helical segments, is conserved among the nine globins even though the level of sequence identity in some pairwise comparisons falls as low as $16 \%$. The latter feature is unusual, since in other protein families, comparably low sequence identities are also accompanied by divergences in secondary structure (Chothia \& Lesk, 1986). In addition, five helix packings occur in the 9 globins, $\mathrm{A} / \mathrm{H}, \mathrm{B} / \mathrm{E}, \mathrm{B} / \mathrm{G}$, $\mathrm{F} / \mathrm{H}$ and $\mathrm{G} / \mathrm{H}$ and it was found that the pattern of contacts between residues at homologous helix interfaces were conserved. However, the superposition of homologous pairs of packed helical segments produced a fit that was generally much worse than for the superposition of individual pairs of homologous helices, with shifts in relative position and orientation ranging to as much as 7 angstroms and 30 degrees, respectively (Lesk \& Chothia, 1980).

Bashford et al. (1987) used the alignment derived by Lesk \& Chothia (1980) to align a total of 226 globin sequences of which only about $10 \%$ were of nonvertebrate origin. Based on the number of occurrences of each amino acid residue type at 10 common homologous positions, they derived two templates diagnostic of the "myoglobin fold". The two temnlates consist of 71 positions and differ only in the scoring of residue substitutions: (1) the 34 positions occupied by interior residues with low, medium and sever restrictions in size; (2) the 32 positions occupied by surface residues, and (3), the 5 positions where any residue is acceptable. Although both templates showed a very substantial ability to discriminate between globins and non-globins, the nonvertebrate globin sequences did not fit the templates very well (i.e., had high scores) and could not always be aligned unambiguously with each other and the vertebrate sequences. The difficulty of avoiding ambiguity in the alignment of two distantly related globins, even though their crystal structures are known, has been amply demonstrated in a recent study of lamprey hemoglobin and 
Aplysia myoglobin, which exhibit only $30 \%$ identity (44 residues out of 138 aligned) (Pastore et al., 1988).

Bordo \& Argos $(1990,1991)$ have used the known crystal structures of vertebrate and nonvertebrate globins to align 135 alpha-like sequences, 130 beta-like sequences and 52 myoglobin sequences and searched the resulting data base for structurally equivalent residue pairs within unmodified environments. The matrices of preferred amino acid exchange constructed for residues within the protein cores and for residues exposed at the protein surface of globins were found to be similar to corresponding matrices constructed for other protein families (Overington et al., 1990, 1992). The buried residue substitutions observed in globins (Bordo \& Argos, 1990), $(\underline{\mathrm{G}}, \underline{\mathrm{A}}), \underline{\mathrm{A}}, \underline{\mathrm{S}}), \underline{\mathrm{A}}, \underline{\mathrm{T}}),(\underline{\mathrm{V}}, \underline{\mathrm{L}}),(\underline{\mathrm{V}}, \mathbf{I}),(\underline{\mathrm{K}}, \underline{\mathrm{L}})$ and $(\underline{\mathrm{L}}, \underline{\mathrm{F}})$, satisfy three diagnostic criteria for amino acid substitutions in a protein core: (a) no more than one methyl group difference in side-chain volume, (b) conservation of side-chain polarity and (c), alteration in radius of gyration of $>0.50$. These criteria are also satisfied by the following amino acid exchanges: $(\mathrm{S}, \mathrm{T}),(\mathrm{S}, \mathrm{P}),(\mathrm{C}, \mathrm{V}),(\mathrm{T}, \mathrm{P})$, $(\mathrm{M}, \mathrm{Y}),(\mathrm{I}, \mathrm{F}),(\mathrm{L}, \mathrm{I}),(\mathrm{F}, \mathrm{Y})(\mathrm{F}, \mathrm{M})$ and $(\mathrm{Y}, \mathrm{W})$. Not surprisingly, the surface residues display much more freedom of alteration than do the buried residues. Depending on the extent of similarity in the residue environment, about 30 to $50 \%$ of surface residue substitutions involve a change in polarity or a change in volume in excess of one methyl group, in contrast to ca. $15 \%$ for buried residues (Bordo \& Argos, 1991).

From 1059 entries in the PIR Data Bank under "globin", we culled 554 unique vertebrate globin sequences and 129 nonvertebrate globin sequences using the criteria enumerated below. The large majority of the nonvertebrate globin sequences have become available in the last five years, together with three additional monomeric globin crystal structures, those of Aplysia 
myoglobin (Bolognesi et al., 1991), Scapharca hemoglobin (Royer et al., 1989) and Urechis hemoglobin (Kolatkar et al., 1992). We have used the secondary structure assignments from the known globin crystal structures and the alignments of Lesk \& Chothia (1980) and of Bashford $e t$ al. (1987), to align all the globin sequences. The unique features of vertebrate and nonvertebrate globins were identified and new globin templates were derived. These templates have been used to evaluate the relationship between the two groups of globin sequences and the recently determined sequences of several heme-binding proteins and putative globins whose alignments range from the ambiguous to improbable. 


\section{Globin Sequences}

All the vertebrate globin sequences were obtained from the Atlas of Protein and Genomic Sequences (National Biomedical Research Foundation), March 31, 1993 release of PIR1, PIR2, PIR3 and PATCHX databases. The 1056 entries were evaluated using the following criteria. (1) All the partial sequences were rejected. Complete globin sequences that were labeled tentative, or contained peptides with undetermined sequences or contained sequenced fragments whose order was not determined experimentally were also eliminated. (2) Where several versions of the same sequence were found, the latest version was used, if different. Although genomic or cDNA sequences were preferred to those determined by chemical methods, the sequences derived from pseudogene sequences were not included. (3) Sequences derived from pseudogene sequences were not included. (4) Sequences identical to the ones already in the list, generally from closely related species, were not included.

The 554 unique vertebrate sequences included 243 alpha-like, 233 beta-like chains and 55 myoglobins sequences. The distribution of the sequences among the principal vertebrate groups is shown in Table 1. It is evident that the mammalian sequences are present in overwhelming proportions. Table 2 shows the biological distribution of nonvertebrate sequences. Table 3 shows the amino acid compositions of vertebrate and nonvertebrate globins and groups of all - $\alpha$ proteins expressed as percent (SD) of total. 
Table 1. Biological distribution of vertebrate globin sequences.

$$
\text { Alpha-like Beta-like Myoglobins Others Total }
$$

$\begin{array}{lrrrrr}\text { Mammals } & 143 & 171 & 56 & - & 370 \\ \text { Birds } & 55 & 31 & - & 2 & 88 \\ \text { Fish } & 17 & 17 & - & 10 & 44 \\ \text { Amphibians } & 22 & 13 & - & - & 35 \\ \text { Reptiles } & 10 & 7 & - & - & 17 \\ \text { Total } & 247 & 239 & 56 & 12 & 554\end{array}$

Table 2. Biological distribution of nonvertebrate sequences.

\begin{tabular}{|c|c|}
\hline Group & $\begin{array}{l}\text { Intracellular } \\
\text { monomeric }\end{array}$ \\
\hline
\end{tabular}

$\begin{array}{lccc}\text { Annelid } & 5^{\mathrm{c}} & 17 & - \\ \text { Molluscan } & 15^{\mathrm{c}} & 11^{\mathrm{c}} & 4 \\ \text { Insect } & - & 32^{\mathrm{c}} & - \\ \text { Echinoderm } & 3^{\mathrm{c}} & - & - \\ \text { Echiuran } & 1^{\mathrm{c}} & - & - \\ \text { Nematode } & - & 4 & 4 \\ \text { Crustacean } & - & - & 11 \\ \text { Protozoan } & - & 3 & - \\ \text { Yeast } & - & - & 2 \\ \text { Algal } & - & 2 & - \\ \text { Bacterial } & - & 2 & 1 \\ \text { Plant } & - & 27^{\mathrm{c}} & - \\ & & 22 \\ \text { Total } & 24 & 98 & \\ & & \end{array}$


Table 3. Amino acid compositions of vertebrate and nonvertebrate globins and groups of all $-\alpha$ proteins expressed as percent (SD) of total.

\begin{tabular}{lccrrrr} 
Amino acid & Verteb & Nonverteb & \multicolumn{3}{c}{ All $-\alpha$ proteins } \\
& & & $A^{\mathrm{b}}$ & $\mathrm{B}^{\mathrm{d}}$ & $\mathrm{C}^{\mathrm{e}}$ & $\mathrm{D}^{\mathrm{f}}$ \\
& $\mathrm{n}=554$ & $\mathrm{n}=108$ & & & $\mathrm{n}=180$ & $\mathrm{n}=36$ \\
Ala & $11.2(2.0)$ & $13.2(3.7)$ & 10.2 & 11.6 & $11.4(4.3)$ & $11.1(4.4)$ \\
Arg & $2.5(0.9)$ & $3.1(1.9)$ & 2.3 & 2.2 & $3.1(2.3)$ & $3.4(2.7)$ \\
Asn & $3.5(1.4)$ & $4.3(1.7)$ & 3.7 & 4.0. & $5.0(1.8)$ & $5.1(2.5)$ \\
Asp & $5.5(1.2)$ & $6.3(1.9)$ & 7.2 & 6.7 & $5.2(2.0)$ & $6.2(2.1)$ \\
Cys & $1.0(0.7)$ & $0.6(0.8)$ & 1.1 & 0.9 & $2.1(1.5)$ & $2.2(2.1)$ \\
Gln & $2.5(1.3)$ & $3.7(1.3)$ & 2.4 & 2.7 & $3.3(1.4)$ & $3.3(1.5)$ \\
Glu & $4.7(2.0)$ & $5.0(2.2)$ & 6.2 & 5.5 & $5.6(1.9)$ & $5.8(2.1)$ \\
Gly & $6.8(1.8)$ & $6.6(2.2)$ & 6.7 & 8.1 & $10.0(2.4)$ & $9.2(2.5)$ \\
His & $6.0(1.5)$ & $2.8(1.5)$ & 4.5 & 4.5 & $1.9(1.4)$ & $2.2(1.8)$ \\
Ile & $2.6(2.2)$ & $4.4(1.6)$ & 3.9 & 3.7 & $4.4(1.8)$ & $4.3(2.2)$ \\
Leu & $11.8(1.6)$ & $9.1(1.8)$ & 9.5 & 9.0. & $7.2(1.9)$ & $7.4(3.1)$ \\
Lys & $8.4(1.8)$ & $7.3(2.5)$ & 11.0. & 10.2 & $10.1(4.7)$ & $8.8(4.1)$ \\
Met & $1.3(0.9)$ & $2.0(1.5)$ & 2.0. & 2.0. & $2.0(1.3)$ & $2.1(1.6)$ \\
Phe & $5.3(0.7)$ & $5.8(1.7)$ & 4.9 & 5.0. & $3.6(1.5)$ & $4.1(1.9)$ \\
Pro & $3.5(1.0)$ & $2.8(1.0)$ & 3.8 & 3.4 & $4.2(2.0)$ & $4.2(2.0)$ \\
Ser & $6.2(2.1)$ & $7.2(2.2)$ & 5.0. & 5.0. & $4.9(2.5)$ & $4.7(2.2)$ \\
Thr & $5.0(1.7)$ & $4.3(1.8)$ & 5.4 & 4.9 & $5.9(1.9)$ & $5.2(1.7)$ \\
Trp & $1.2(0.7)$ & $1.7(0.5)$ & 1.3 & 1.3 & $1.0(0.7)$ & $1.2(0.8)$ \\
Tyr & $2.2(0.8)$ & $1.8(0.9)$ & 2.7 & 2.6 & $4.2(1.4)$ & $4.2(1.6)$ \\
Val & $8.8(2.4)$ & $7.8(2.1)$ & 6.2 & 6.8 & $4.9(2.1)$ & $5.2(1.9)$ \\
& & & & & &
\end{tabular}

- Calculated from 554 vertebrate hemoglobins and myoglobin sequences.

${ }^{b}$ Calculated from 108 monomeric nonvertebrate globin sequences.

- 16 all alpha proteins, including 6 vertebrate globins (Cid et al., 1992).

d 19 all alpha proteins, including 8 vertebrate and 2 nonvertebrate globins (Chou \& Zhang, 1992).

- Calculated from the sequences of 12 hemerythrins and myohemerythrins, 75 cytochromes $c, 10$ cytochromes $c_{2}, 3$ cytochromes $c_{550}, 9$ cytochromes $c_{551}, 17$ cytochromes $c_{553}, 30$ phycocyanins, 6 phycoerythrocyanins, 3 apoferritins and 9 growth factors taken from the PIR.

${ }^{\mathrm{f}}$ Calculated from 3 sequences of each of the 12 groups above. 
A total of 143 nonvertebrate globin sequences were used, including 128 from the PIR database and an additional 15 recently determined sequences. The latter include Nassa myoglobin (Parente et al., 1993), two globins of Tokunagayusirika (Fukuda et al., 1993), one and two-domain globins of Barbatia virescens and B. lima (Suzuki et al., 1992a, 1993), Liolophura (Suzuki et al., 1992b) myoglobin, the globin chains of the vestimentiferen Oligobrachia (T. Suzuki, unpublished observations) and two Lucina globins (Hockenhull-Johnson et al., 1991, 1993). Of the 143 globins, 114 were monomeric globins from the following phyla: 22 annelid, 26 mollusc, 27 plant, 32 insect, 3 echinoderm, 2 nematode, and one each of echiuran and bacterial origin. The remaining sequences consisted of 5 truncated globins of $<130$ amino acid residues, 8 globin-like domains from chimeric two-domain chains, 11 globin-like domains from chimeric, multi-domain chains and 3 globin-like domains from chimeric proteins containing an additional protein domain unrelated to globins. The data set used in this work is available upon request. 


\section{Alignment of Globin Sequences}

The following guidelines were used in the alignment. (1) Globins belonging to a group in which one member has a known crystal structure, were assumed to have an identical secondary structure. It should be noted that although the "myoglobin fold" persists in the known crystal structures of monomeric vertebrate and nonvertebrate globins, the boundaries of the alpha-helical segments are not constant. (2) The length of a given interhelical segment was defined by the number of positions between the shortest helices found among the known crystal structures. Thus, for example, the A-B interhelical region occupied positions 27 to 40 , since the A helix of Scapharca is the shortest A helix, stopping at position 27 and the B helix of Chir.momus HbIII is the shortest B helix, beginning at position 40 (instead of 36 or 37 for the other structures). (3) All interhelical regions were expanded just enough to accommodate the longest known sequences in these regions. Thus, for example, the A-B interhelical region is defined by the sequences of Lumbricus chain III (Fusitani et al., 1988) and Lamellibrachia chain BIV (Suzuki et al., 1991). (4) The residues comprising the helical regions were aligned with the sperm whale myoglobin sequence. Because the presence of a $D$ helix in the alpha-chain and its absence from the beta-chain was found not to affect the properties of human hemoglobin (Komiyama et al., 1992), we made no assumption of its presence in the C-E interhelical region. (5) The interhelical sequences were aligned heuristically, following as much as possible the allowed amino acid exchanges for globin surface residues (Bordo \& Argos, 1991).

The 182 positions used in our alignment are greater than the 171 positions used by Lesk \& Chothia (1980). The difference is due to insertions found in the nonvertebrate sequences in the A - B, E - F and F - G interhelical regions. Figure 1 shows our alignment of the globin sequences that defines the 182 positions. Of these, 96 positions were common to $99 \%$ of both the vertebrate 
and the monomeric nonvertebrate groups of sequences, 586 out of 592 and 114 of 115 , respectively. The number of common positions were 112 at the $90 \%$ level (533 and 104, respectively) and 124 at the $75 \%$ level ( 444 and 86 , respectively).

Figure 2 shows histograms of lengths of the subgroup sequences. In the second histogram, the open rectangles indicate the invertebrate myoglobins. 


\section{Analysis of the Aligned Sets of Globins}

\section{(a) Vertebrate globins and representational equivalence}

In our experience, the usefulness of the Needleman-Wunsch algorithm for pairwise alignment of globin sequences is somewhat limited, as noted earlier by Bashford et al. (1987). In the case of globins whose three-dimensional structures are known from crystallographic studies, the resulting alignments frequently run counter to the alignments necessitated by the known secondary structures. The alignments of Lesk \& Chothia (1980) and of Bashford et al. (1987) which are predominantly based on the maintenance of the secondary structure provide in our opinion, the most logical approach towards the alignment of globin sequences. In this sense, our alignment is an extension of their approach.

Figure 3 shows the amino acid substitution profile of the 554 vertebrate globins, including 247 alpha-like, 239 beta-like globins and 56 myoglobins, at the 138 positions (out of 18) that are common to $98 \%$ of the vertebrate sequences: 1 through 27 (NA2 - A15), 36 through 62 (B1 through CD4), 65 through 67 (CD7-CD9), 73 through 95 (D6-EF1), 97 (EF3), 99 through 103 (EF5_EF9), 109 through 120 (F10FG2), 124 and 126 (FG6 and FG8), 127 through 145 (G1-G19) and 147 through 175 (GH2-H24). At each position, the ascending order of residues corresponds to the decreasing order in frequency of occurrence.

(b) Features of vertebrate sequences

Only one sequence has a proximal residue that is neither His nor Gln: The bovine epsilon-2 chain has a Tyr. (Schimenti \& Duncan, 1985).

(c) Alignment of Nonvertebrate globins 
The nonvertebrate globins display a much greater variation in their covalent structure than do vertebrate globins. The 143 sequences can be divided into the following groups (Vinogradov et al., 1993): (1) 116 monomeric globins, (2) 6 truncated globins consisting of 116 to 125 amino acid residues, (3) 3 globin domains from chimeric chains containing one globin domain and one non-globin domain, (4) 8 globin domains from chimeric chains comprising two linearly and covalently linked globins and (5), 11 globin domains derived from the chimeric multi-domain chains of Artemia extracellular hemoglobin comprising 9, linearly and covalently linked globin domains. It should be noted that in addition, many of the above five classes of globin chains participate in a broad variety of quaternary structure, ranging from homo and heterodimers to highly complex, multisubunit aggregates with $\mathrm{Mr}>3,500 \mathrm{kDa}$ (Vinogradov, 1985).

The monomeric nonvertebrate globins exhibit the widest distribution and span a somewhat broader range of polypepetide chain lengths than do the vertebrate globins as seen in Figure 2 . The intracellular monomeric globins include the monomeric and polymeric Hbs of the polychaete Glycera, the tetrameric $\mathrm{Hb}$ of the echiuran Urechis, the dimeric Hbs of echinoderms such as Paracaudina and Caudina (Molpadia) and the dimeric and tetrameric Hbs of the bivalve molluscs Scapharca, Anadara, Barbatia and Calyptogen. The extracellular monomeric globins include the multiple monomeric and dimeric Hbs of the larva of the insect Chironomus, the hemoglobins of nematodes such as Trichostrongylus and the globin chains forming $\sim 2 / 3$ of the highly complex, giant hexagonal bilayer hemoglobins and chlorocruorins of annelids such as Lumbricus and Tylorrhynchus and vestimentiferans, such as Lamellibrachia. The cytoplasmic Hbs include the myoglobins of he mollusc Aplysia, the three hemoglobins o the symbiont-harboring clanı Lucina, the hemoglobin of the bacterium Vitreoscilla and plant hemoglobins, including the 
leghemoglobins of symbiont-containing legumes, the globins found in the roots of symbiont-free plants and the globins of symbiont-containing non-luguminous plants. Furthermore, the eight known crystallographic structures of nonvertebrate globins are all of monomeric chains. 
Figure 1. The sequences of sperm whale myoglobin and of nonvertebrate globins, of known crystal structure, Glycera, Lupinus, Chironomus, Aplysia, Scapharca and Urechis and of those which define the 182 positions: Paracaudina, Lumbricus, Liolophura, Barbatia and Vitreoscilla.



a The Mb fold includes Template I of Bashford et al. (1987) which consists of 71 positions: (1) 34 interior positions with low, nedium and sevens restrictions in size according to Table 4 of Bashford et al. (1987) are indicated by lower case, lower case boldeace and cApIrne Boidracs, respectively $;$ in all the sequences, these residues are also in boldiace; (2) the 32 positions occupied by surface residues are underlined; (3) the 5 positions where any residue is acceptable according to Template I (B5, EF 1, FG2, G19 and H2O) are indicated by a boldface X. The distal and proximal residues are indicated by $D$ and $P$, respectively. 
HISTOGRAMS OF SUBGROUP SEQUENCE LENGTHS
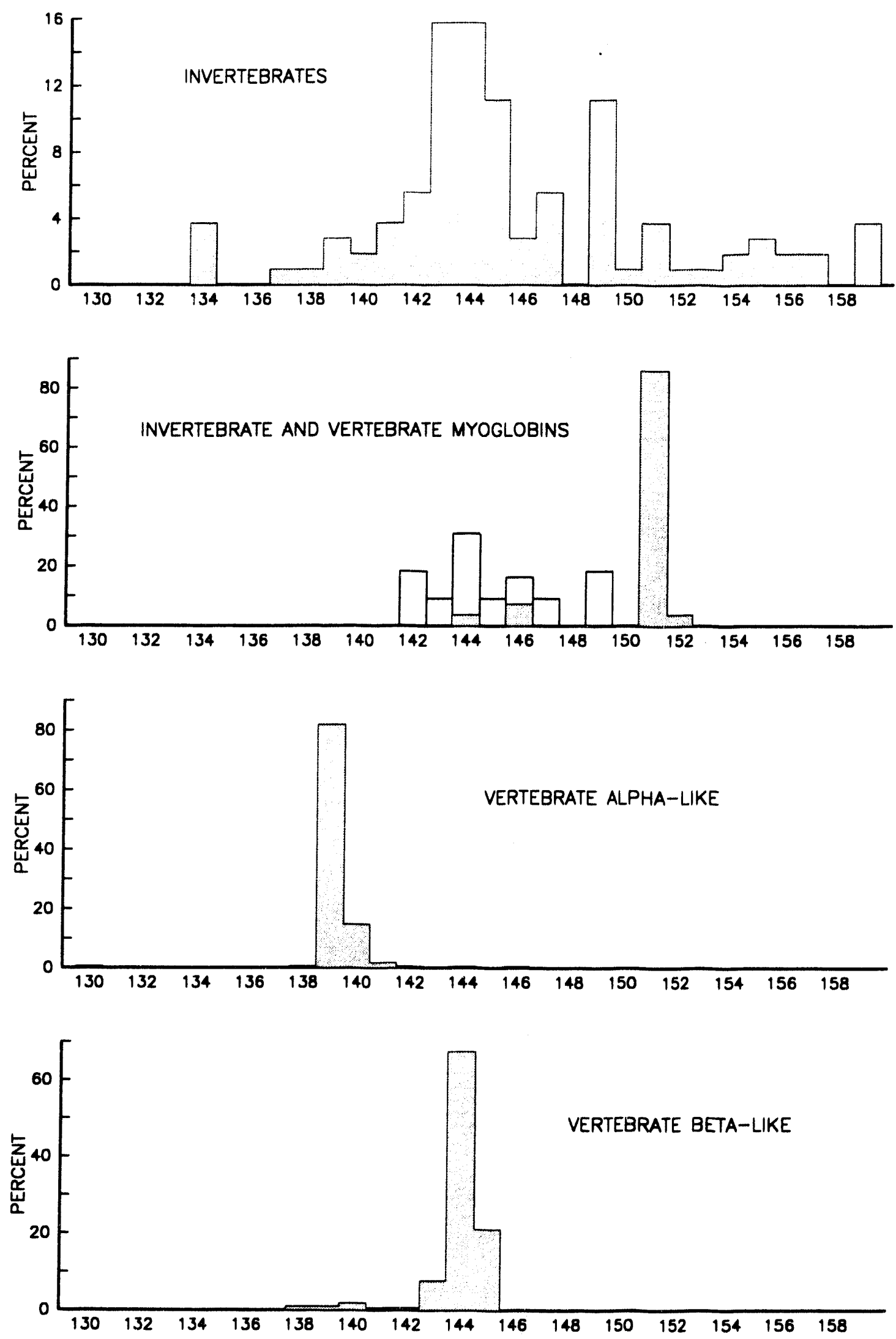
Fig. 3

1 OCCURANCE
O

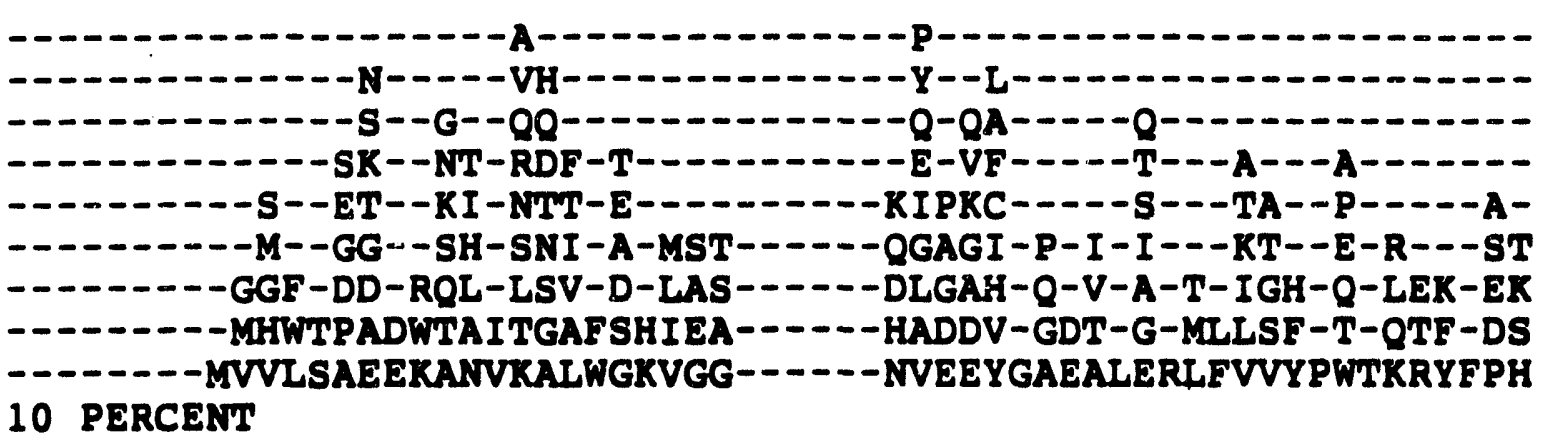

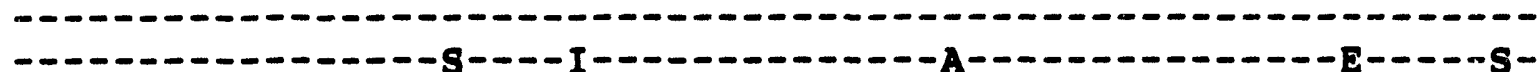



- - - - - HWTPAD-TAITGA-S-IEA-- - - HADDV-G-T-G-MILSF-T-QTF-DS

--.----VVLSAEERAIVKALWGKVGG-----NVEEYGAEALERLFVVYPWTKRYFPH

20 PERCELT

-

-------VVLSAEERANVKALWGKVGG------NVEEYGAEALERLFVVYPWTKRYF PH

50 PERCENT

- - - - - - - LS- - EX--V- - WGKV

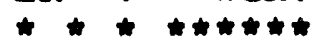

anaAaAnaAaAnaAa

1234567891111111

0123456
-G-EAL-RLF- YP-T- YF--

$\star \star \star \star \star * \star \star \star * \star$

BBBBBBBBBBBBBBBBCCCCCCCCCC $12345678911111111234567 D D D$

0123456 
Fig. 3 (cont.)
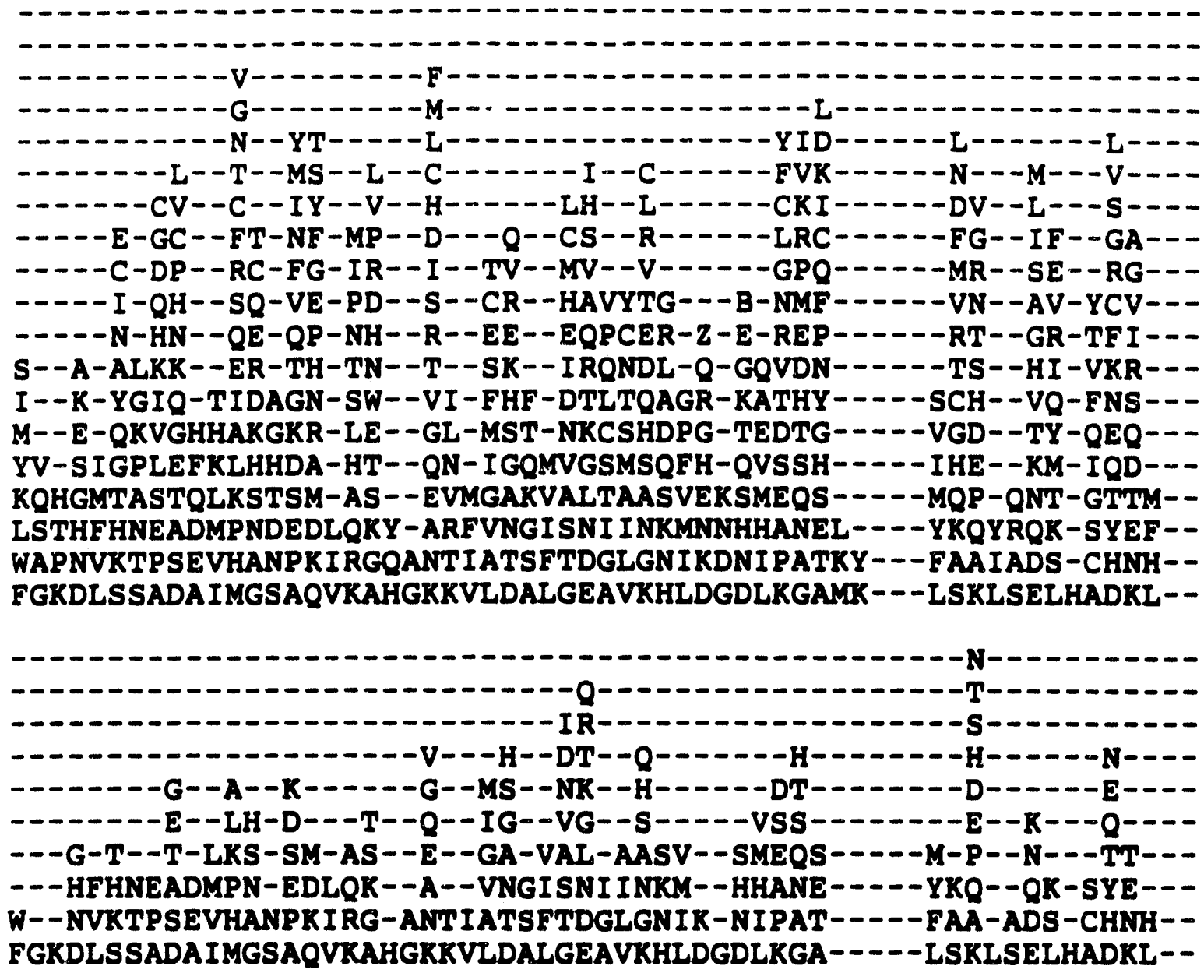

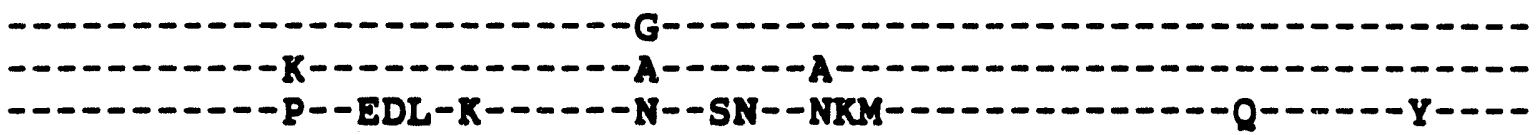
---N-DPS-VH-NPKIRG---T-ATSFTDGLGNI- -NIPAT---- FAA-AD--CH-H-FGKDLSSADA IMGSAQVKAHGKKVLDALGEAVKHLD-DLKGA-----LSKLSELHADKL--

$-\mathbf{s - 2}$

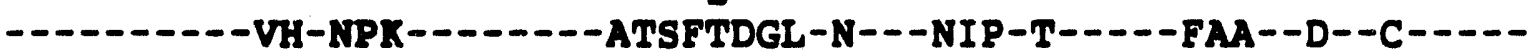
FG-DLSSA-AIMGSAQVKAHGKKVLDALGEAVKHLD-DLKGA-----LSKLSELHADKL--

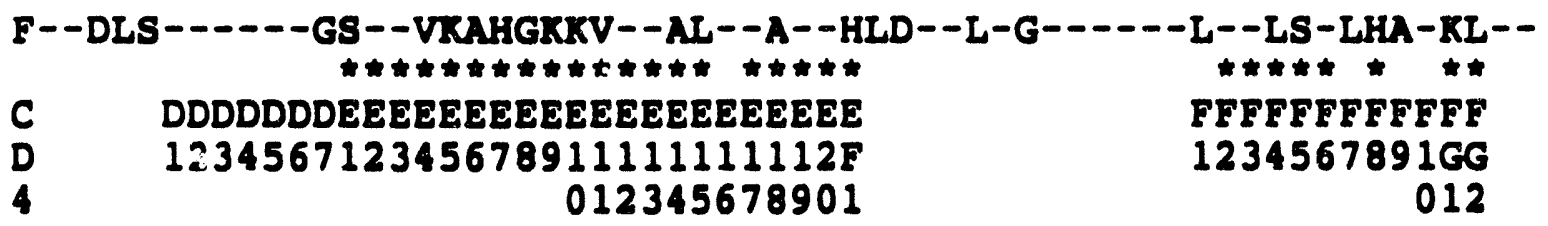


Fig. 3 (cont.)



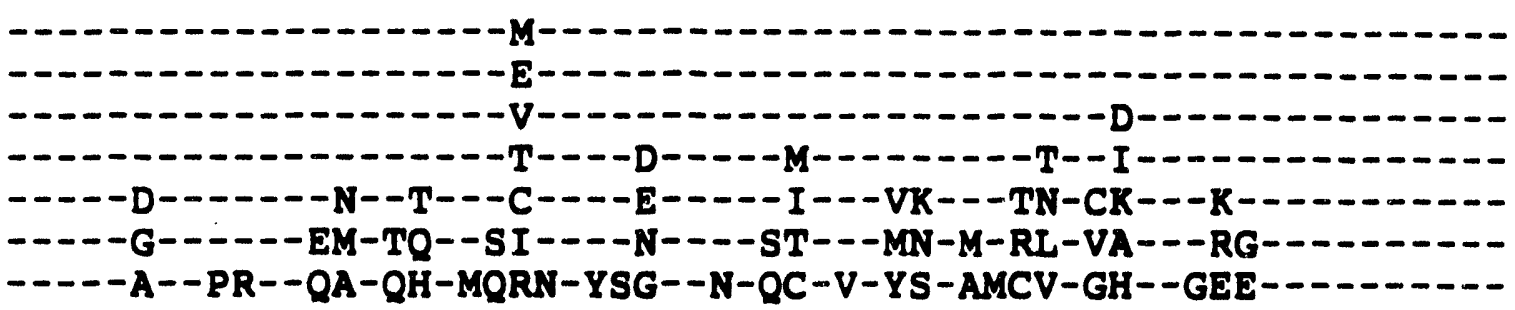

K---VK--EI-ADIF ICI IHSR-LASAYS-DL-S-FE-LFESIRSDMSAQ-K--.-- R-IPIVYLRF ISNVIVITVGHK-HGADLGAAAHGSWQ-VVSAFSNVITHR-H--- -H--H-VDPENFKLLGHCLLVVLAAH-FPKEFTPEVQAALDKFLAGVATALASKYRELGFQG--

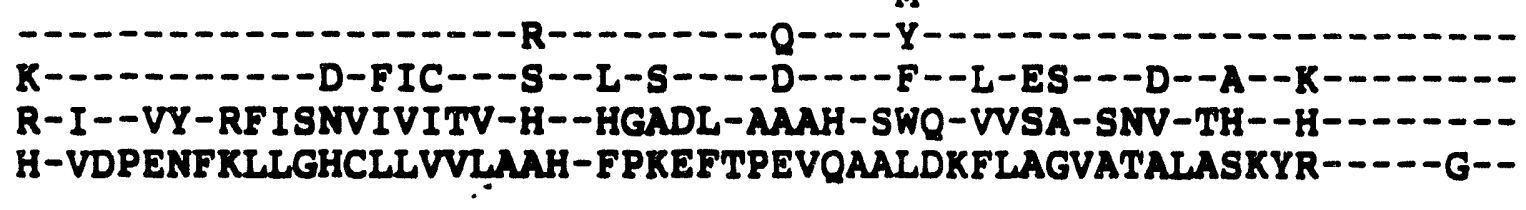

R----V--R--SNVIV-T-D- - HGAD- -A-H-S-O-VV-A-SNV-TH- HH-VDPENEKLLGHCLLVVLAAH-FPKEFTPEVQAALDKFLAGVATALASKYR--.---

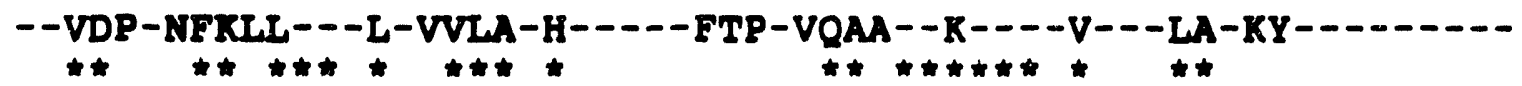

F FGGGGGGGGGGGGGG

G G1234567891111111111

340123456789

НHНHНHНHНHНHНHНHНHНHНHНHНH

12345678911111111112222222

01234567890123456 


\section{References}

Arents, G. \& Love, W.E., Glycera dibranchiata hemoglobin: structure and refinement at 1.5 angstroms. J. Mol Biol. 210, 149-161 (1990).

Arutyunyan, E.G., Kuranova, I.P., Vainshtein, B.K. \& Steigemann, W., X-ray structural investigation of leghemoglobin. VI. Structure of acetate-ferrileghemoglobin at a resolution of 2.0 angstroms. Sov. Phys. Crystallogr. 25, 43-56 (1980).

Arutyunyan, E.G., The structure of leghemoglobin. Molek. Biol. 15, 27-44 (1981).

Bashford, D., Chothia, C. \& Lesk, A.A., Determinants of a protein fold: unique features of the globin amino acid sequences. J. Mol. Biol. 196, 199-216 (1987).

Bolognesi, M., Onesti, S., Gatti, G., Coda, A., Ascenzi, P. \& Brunori, M., Aplysia limacina myoglobin: crystallographic analysis at 1.6 angstrom resolution. J. Mol. Biol. . 205, 529-544 (1989).

Bordo, D. \& Argos, P., Evolution of protein cores: constraints in point mutation as observed in globin tertiary structure. J. Mol Biol. 211, 975-988 (1990).

Bordo, D. \& Argos, P., Suggestions for "safe" residue substitutions in site-directed mutagenesis. J. Mol. Biol. 217, $721-729$ (1991).

Braunitzer, G., Gehring-Muller, R., Hilschmann, N., Hilse, K., Hobom, G., Rudloff, V. \& Wittmann-Liebold, B., Z. Physiol. Chemie 325, 283-286 (1961).

Carson, W.M., Bowers, T.R., Kitto, G.B. \& Hackert, M.L., Preliminary crystallographic data on monomeric and dimeric hemoglobins from the sea cucumber Molpadia arenicola. J. Biol. Chem. 254, 7400-7402 (1979).

Chiacone, E., Vecchini, P., Verzili, D., Ascoli, F \& Antonini, E., Dimeric and tetrameric hemoglobins from the mollusc Scapharca inaequivalvis J. Mol. Biol. 152, 577-592 (1981).

Chiancone, E., Verzili, D., Boffi, A., Royer Jr., W.E., \& Hendrickson, W. A., Scapharca inaequivalvis hemoglobins: novel cooperative assemblies of globin chains. In Structure and Function of Invertebrate Oxygen Carriers (Vinogradov, S.N. \& Kapp, O.H., eds.), pp. 139-145, Springer Verlag, New York (1991).

Dixon, B., Walker, B., Kimmins, W. \& Pohajdak, B., Isolation and sequencing of an unusual hemoglobin from the parasitic nematode Pseudoterranova decipiens. Proc. Natl. Acad. Sci. U.S. 88, 5655-5659 (1991).

Edmundson, A.B., Amino acid sequence of sperm whale myoglobin. Nature 205, 883-890 (1965). 
Fermi, G. \& Perutz, M.F., Haemoglobin and Myoglobin, Clarendon Press, Oxford (1981).

Fukuda, M., Takagi, T. \& Shikama, K., Polymorphic hemoglobin from a midge larva (Tokunagayusurika akamusi) can be divided into two different groups. Biochim. Biophys. Acta (In press) (1993).

Fushitani, K., Matsuura, M.S.A. \& Riggs, A.F., The amino acid sequences of chains a, b and c that form the trimer subunit of the extracellular hemoglobin of Lumbricus terrestris. J. Biol. Chem. 263, 6502-6517 (1988).

Gilles-Gonzalez, M.A., Ditta, G.S. \& Helinski, D.R., A haemoprotein with kinase activity encoded by the oxygen sensor of Rhozobium meliloti. Nature 350, 170-172 (1991).

Gagne, G. \& Guertin, M., The early genetic response to light in the green unicellular alga Chlamydomonas eugametos grown under light/dark cycles involves genes that represent direct responses to light and photosynthesis. Plant Mol. Biol. 18, 429-445 (1992).

Hockenhull-Johnson, J.D., Stern, M.S., Martin, P., P., Dass, C., Desiderio, D.M., Wittenberg, J.B., Vinogradov, S.N. \& Walz, D.A., The amino acid sequence of hemoglobin II from the symbiont-harboring clam Lucina pectinata. J. Prot. Chem. 6, 609-622 (1991).

Hockenhull-Johnson, J.D., Stern, M.S., Wittenberg, J.B., Kapp, O.H., Vinogradov, S.N. \& Walz, D.A., The amino acid sequence of hemoglobin III from the symbiont-harboring clam Lucina pectinata. J. Prot. Chem. 12, 261-277 (1993).

Holm, L. and Sander, C., Structural alignment of globins, phycocyanins and colicin A. FEBS Lett. 315, 301-305 (1993).

Huber, R., Epp, O. \& Formanek, H., Structures of deoxy- and carbonmonoxy-erythrocruorin. J. Mol. Biol. 52, 349-354 (1970).

Huber, R., Epp, O., Steigemann, W. \& Formanek, H., The atomic structure of erythrocruorin in the light of the chemical sequence and its comparison with myoglobin. Eur. J. Biochem. 19, 42.50 (1971).

Iwaasa, H., Takagi, T. \& Shikama, K., Protozoan myoglobin from Paramecium caudatum. J. Mol. Biol. 208, 355-358 (1989).

Iwaasa, H., Takagi, T. \& Shikama, K., Protozoan hemoglobin from Tetrahymena pyriformis. J. Biol. Chem. 265, 8603-8609 (1990).

Iwaasa, H., Takagi, T. \& Shikama, K., Amino acid sequence of hemoglobin from yeast Candida norvegensis. Zool. Sci. 8, 1134 (1991). 
Kendrew, J.C., Dickerson, R.E., Strandberg, B.E., Hart, R.G., Davis, D.R., Phillips, D.C. \& Shore, V.C., Nature 185, $422-427$ (1960).

Kolatkar, P.R., Meador, W.E., Stanfield, R.L. \& Hackert, M., Novel subunit structure observed for noncooperative hemoglobin from Urechis caupo. J. Biol. Chem. 263, 3462-3465 (1988).

Lolatkar, P.R., Hackert, M.L. \& Riggs, A.F., The structural analysis of Urechis caupo hemoglobin. J. Biol. Chem. (in press) (1992).

Komiyama, N.H., Shih, D.T., Looker, D., Tame, J. \& Nagai, K., Was the loss of the D helix in alpha globin a functionally neutral mutation? Nature 352, 349-351 (1991).

Lesk, A.M. \& Chothia, C., How different amino acid sequences determine similar protein structures: the structure and evolutionary dynamics of the globins. J. Mol. Biol. 136, 225-270 (1980).

Lesk, A.M., Levitt, M. \& Chothia, C., Protein Eng. 1, 77-78 (1986).

Lim, V.I. \& Ptitsyn, O.B., Constancy of hydrophobic nucleus volume in myoglobin and hemoglobin molecules. Mol. Biol. (USSR) 4, 295-303 (1970).

Manning, A.M., Trotman, C, and Tate, W.P., Evolution of a polymeric globin in the brine shrimp Artemia. Nature 348, 653-656 (1990).

Ollis, D., Appleby, C., Colman, P., Cutten, A., Guss, J. \& Freeman, H., Crystal structure of soybean ferric leghaemoglobin a nicotinate at a resolution of 3.3 angstroms. Aust. J. Chem. 36, 451-468 (1983).

Padlan, E.A. \& Love, W.E., Three-dimensional structure of hemoglobin from the polychaete annelid Glycera dibranchiata at 2.5 angstroms. J. Biol. Chem., $4067-4078$ (1974).

Parente, Biochim. Biophys. Acta. (in press) (1993).

Pastore, A., Lesk, A.M., Bolognesi, M. \& Onesti, S., Structural alignment and analysis of two distantly related proteins: Aplysia limacina myoglobin and sea lamprey globin. Proteins Struc. Func. Genet. 4. 240-250 (1988).

Pastore, A \& Lesk, A.M., Comparison of the structures of globins and phycocyanins: evidence for evolutionary relationship. Proteins Struct. Func. Genet. 8, 133-155 (1990).

Perutz, M.F., Rossman, M.G., Cullis, A.F., Muirhead, H., Will, G \& North, A.C.T., Structure of haemoglobin. A three-dimensional Fourier synthesis at 5.5 angstroms resolution, obtained by $\mathrm{x}$-ray analysis. Nature 185, 416-422 (1960). 
Perutz, M.F., Kendrew, J.C. \& Watson, H.C., Structure and function of haemoglobin. J. Mol. Biol. 13, 669-678 (1965).

Perutz, M.F., Fermi, G., Luisis, B. \& Liddington, R.C., Stereochemistry of cooperative effects in hemoglobin. Accts. Chem. Res. 20, 309-317 (1987).

Phillips, S.E.V., Structure and refinement of oxymyoglobin at 1.6 angstroms resolution. J.Mol. Biol. 14, 53 1-554 (1980).

Potts, M., Angeloni, S.V., Ebel, R.E. \& Bassam, D., Myoglobin in a cyanobacterium. Science 256, 1690-1692 (1992).

Probst, L., Wolf, G. \& Schlegel, H.G., An oxygen binding flavohemoprotein from Alcaligenes eutrophus. Biochim. Biophys. Acta 576, $471-478$ (1979).

Ptitsyn, O.B., Invariant features of globin primary structure and coding of their secondary structure. J. Mol. Biol. 88, 287-300 (1974).

Romero-Herrera, A.E., Lehmann, H., Joysey, K.A. \& Friday, A.E., Phil. Trans. Roy. Soc. 283, $61-83$ (1978).

Royer Jr, W.E., Love, W.E. \& Henderson, F.F., Cooperative dimeric and tetrameric clam hemoglobins are novel assemblages of myoglobin folds. Nature, 316, 277-280 (1985).

Royer Jr., W.E., Hendrickson, W.A. \& Chiancone, E., Structural transitions upon ligand binding in a cooperative dimeric hemoglobin. Science 249, 518-521 (1989).

Runnegar, B., Derivation of the globins from type b cytochromes. J. Mol. Evol. 21, 33-41 (1984).

Schimenti, J.C. \& Duncan, C.H., Concerted evolution of the cow epsilon-2 and epsilon-4 beta-globin genes. Mol. Biol. Evol. 2, 505-513 (1985).

Steigemann, W \& Weber, E., Structure of erythrocruorin in different ligand states refined at 1.4 angstroms resolution. J. Mol. Biol. 127, 309-338 (1979).

Suzuki, T. \& Takagi, T., A myoglobin evolved from indoleamine 2,3- dioxygenasee. J. Mol. Biol. 228, 698-700 (1992).

Suzuki, T., Takagi, T \& Ohta, S., Amino acid sequence of a dimeric haemoglobin from the deep-sea clam Calyptogena soyoae. Biochem. J. 260, 177-182 (1989b).

Suzuki, T., Takagi, T. \& Ohta, S., Amino acid sequence of the dimeric hemoglobin (HbI) from the deep-sea cold-seep clam Calyptogena soyoae and the phylogenetic relationship with other molluscan globins. Biochim. Biophys. Acta 999, 254-259 (1989c). 
Suzuki, T., Takagi, T. \& Ohta, S., Primary structure of constituent polypeptide chain (AIII) of the giant haemoglobin from the deep-sea tubeworm Lamellibrachia. Biochem. J. 266, 221-225 (1990b).

Suzuki, T., Nakamura, A., Satoh, Y., Inai, C., Furukohri, T. \& Arita, T., Primary structure of chain I of the heterodimeric hemoglobin from the blood clam Barbatia virescens. J. Prot. Chem. 11, 629-633 (1992).

Takagi, T., Iwaasa, H., Ohta, S. \& Suzuki, T., Primary structure of $440 \mathrm{kDa}$ hemoglobin from the deep sea tubeworm Lamellibrachia. In: Strucure and Function of Invertebrate Oxygen Carriers (Vinogradov, S.N. \& Kapp, O.H., eds) pp. 245-249, Springer Verlag, New York (1991).

Trotman, C.N.A., Manning, A.M., Moens, L., Guise, K.J. \& Tate, W.P Translation of the cDNA sequence for the polymeric hemoglobin of Artemia. In: Structure and Function of Invertebrate Oxygen Carriers (Vinogradov, S.N.\& Kapp, O.H., eds) pp. 207-216, Springer Verlag, New York (1991).

Vasudevan, S.G., Armarego, W.L.F., Shaw, D.C., Lilley, P.E., Dixon, N.E. \& Poole, R.K., Isolation and nucleotide sequence of the hmp gene that encodes a haemoglobin-like protein from Escherischia coli K-12. Mol. Gen. Genet. 226, 49-58 (1991).

Wakabayashi, S., Matsubara, H. \& Webster, D.A., Primary sequence of a dimeric bacterial haemoglobin from Vitreoscilla. Nature 322, 481-483 (1986).

Weihs, V., Schmidt, K., Schneider, B \& Friedrich, B., The formation of an oxygen-binding flavohemoprotein in Alcaligenes eutrophus. Arch. Microbiol. 151, 546-550 (1989).

Zharkikh, A.A., Solovyov, V.V. \& Kolchanov, N.A., Conformational changes in the globin family during evolution. I. Analysis of the evolutionary role of insertions and deletions. J. Mol. Evol. 21, 42-53 (1984).

Zhu, H. \& Riggs, A.F., Yeast flavohemoglobin is an ancient protein related to globins and a reductase family. Proc. Natl. Acad. Sci. 89, 5015-5019 (1992). 


\section{Recent Publications}

\section{2}

C.-T. Chen, O.H. Kapp and W.H. Wong, Stochastic reconstruction of incomplete data sets using gibbs priors in positron emission tomography. SPIE Proc. on Biomedical Image Processing and Three-Dimensional Microscopy, 26-29 (San Jose, CA, 1992).

O.H. Kapp and C.-T. Chen, Reconstruction from limited projection data. SPIE Symposium on Electronic Imaging: Science and Technology (San Jose, CA, Feb. 9-14, 1992).

O.H. Kapp, Use of an APL interface on a RISC workstation. SPIE Proc., 8 (Chicago, IL, March 19, 1992).

S. Ruan and O.H. Kapp, Scan system for the sextupole-corrected scanning transmission electron microscope. Rev. Sci. Instr. 63, $4066-4070$ (1992).

A.V. Crewe, Electron motion in tuned fields, I. The algebra. Ultramicroscopy 41, 269-277 (1992).

A.V. Crewe, Electron motion in tuned fields, II. Some applications. Ultramicroscopy 41, 279-285 (1992).

A.V. Crewe, A.D. Feinerman, D.A. Crewe, D.C. Perng and S.E. Shoaf, Sub-centimeter micromachined electron microscope. J. Vac. Sci. Technol. A 10(4), 611-616 (1992).

O.H. Kapp and C.-T Chen, Reconstruction from limited projection data. SPIE Proceedings on Biomedical Image Processing and Three-Dimensional Microscopy 1661, 26-29 (San Jose, CA, Feb. 9-14, 1992).

C.-T. Chen, O.H. Kapp and W.H. Wong, Stochastic reconstruction of incomplete data sets using Gibbs priors in positron emission tomography. SPIE Symposium on Electronic Imaging: Science and Technology 1661, 131-134 (San Jose, CA, Feb. 9-14, 1992).

O. Kapp and S. Ruan, Analysis of STEM images on a RISC workstation with an APL interface. SPIE Proceedings: Imaging Technologies and Applications 1778, 117-119 (1992).

S. Ruan and O. Kapp, Scan system for the sub-angstrom STEM. SPIE Proceedings: Imaging Technologies and Applications 1778, 99-102 (Chicago, II, March 19, 1992).

An automatic field emission tip conditioning system. SPIE Proceedings: Imaging Technologies and Applications 1778, 117-119 (1992). 
S. Ruan and O.H. Kapp, An automatic field emission tip conditioning system. Rev. Sci. Instr. 63, 4056-4060 (1992).

S. Ruan and O.H. Kapp, Progress on the sextupole-corrected sub-angstrom resolution scanning transmission electron microscope. SPIE Mtg. (Chicago, IL, March 19, 1992).

A.V. Crewe, A.D. Feinerman, D.A. Crewe, D.C. Perng and S.E. Shoaf, Microfabrication of arrays of scanning electron microscopes. J. Vac. Sci. Technol. (in press).

\section{3}

A.V. Crewe, Electron microscope of the 21 st century. Proc. MSA, 2-3 (Cincinnati, 1993).

J.D. Hockenhull-Johnson, M.S. Stern, J.B. Wittenberg, S.N. Vinogradov, O.H. Kapp, and D.A. Walz, The amino acid sequence of hemoglobin III from the symbiont-harboring clam Lucina pectinata. J. Prot. Chem. 12, 261-277 (1993).

S.N. Vinogradov, D.A. Walz, B. Pohajdak, L. Moens, O.H. Kapp, T. Suzuki and C.N.A. Trotman, Adventitious variability? The amino acid sequences of nonvertebrate globins. Comp. Biochem. Physiol. 106b, 1-26 (1993)

S. Ruan and O.H. Kapp, Tip alignment system in a sextupole-corrected scanning transmission electron microscope, Rev. Sci. Instr. 64, 667-671 (1993).

O.H. Kapp and J. Mukherjee, Modeling of receptor proteins using homology: Implications for nuclear medicine imaging. 4Ist Annual Mtg., Assoc. of Univ. Radiologists. May 19-23, 1993, Cincinnati, Ohio.

O.H. Kapp and C.-T. Chen, Reconstruction techniques from limited projection data. 41st Annual Mig., Assoc. of Univ. Radiologists. May 19-23, 1993, Cincinnati, OH.

C.-T. Chen, O.H. Kapp, W.H. Wong, Stochastic reconstruction of incomplete data sets using Gibbs priors in positron emission tomography. 4lst Annual Mtg., Assoc. of Univ. Radiologists. May 19-23, 1993, Cincinnati, OH.

J. Mukherjee and O.H. Kapp, Selective radiotracers for imaging dopamine D-2 receptors by PET: Molecular nature of receptor-radiotracer interaction. 41st Annual Mtg., Assoc. of Univ. Radiologists. May 19-23, 1993, Cincinnati, OH.

A.V. Crewe, The aberration problem in electron optics. Proc. SPIE, 77-84 (San Diego, 1993).

A.V Crewe, Computer simulation of a corrected STEM. Journ. Micr. (1993) (in press).

S.N. Vinogradov, D.A. Walz, B. Pohajdak and O.H. Kapp, The organization of nonvertebrate globin genes. Comp. Biochem. Physiol. (1993) (in press). 
O.H. Kapp, Some improvements to the IRT algorithm for limited projection data sets. Optik (1993) (submitted).

O.H. Kapp, L. Moens, C.N.A. Trotman, T. Suzuki and S.N. Vinogradov, What is a Globin? Identity profiles of vertebrate and nonvertebrate globins. J. Mol. Biol. (1993) (submitted). 

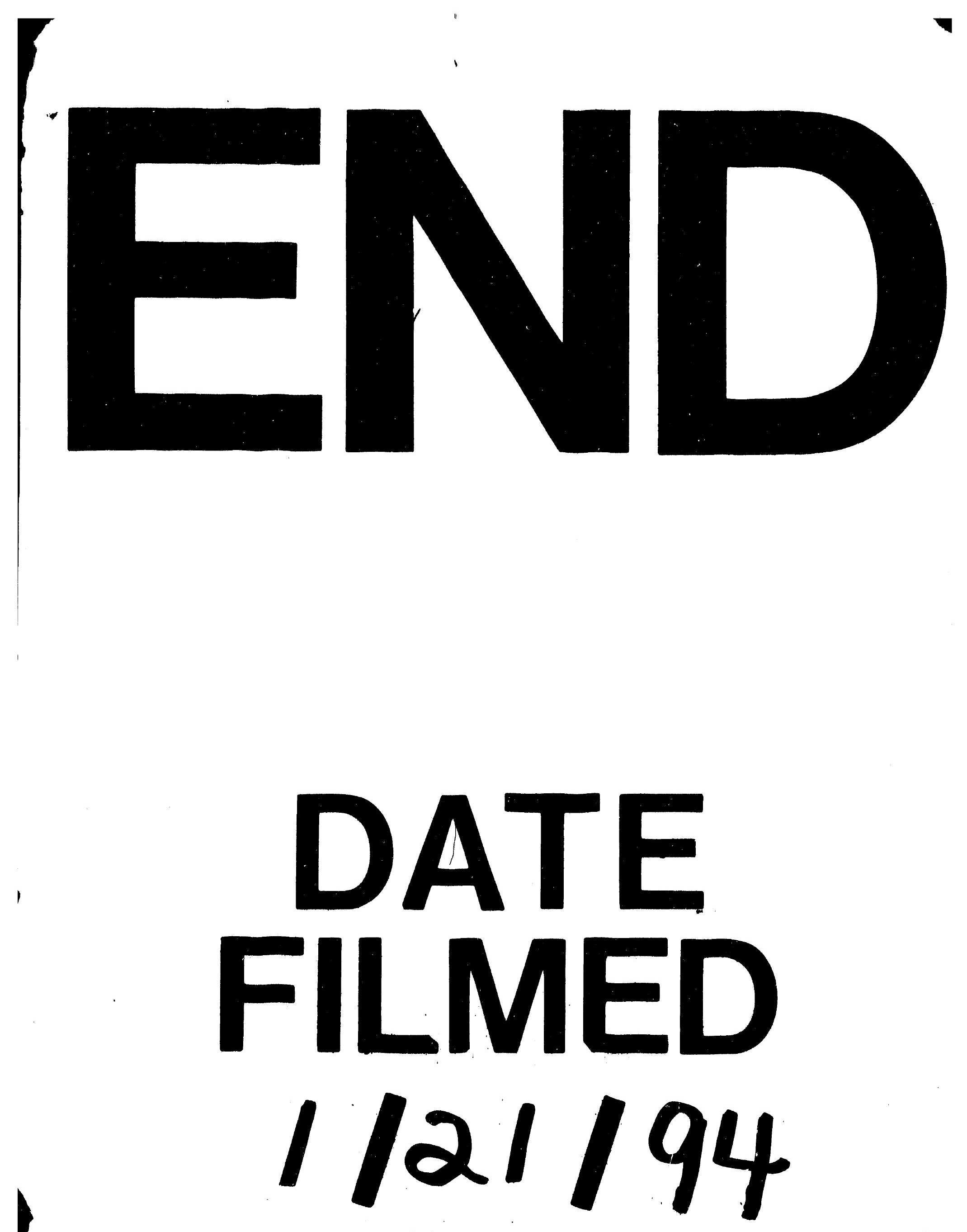
